\title{
Combining phase field approach and homogenization methods for modelling phase transformation in elastoplastic media
}

\author{
Kais Ammar* - Benoît Appolaire** — Georges Cailletaud* \\ Samuel Forest* \\ * Mines ParisTech, Centre des Matériaux/CNRS UMR 7633 \\ BP 87, F-91003 Evry cedex \\ $\{$ kais.ammar, georges.cailletaud, samuel.forest\}@ensmp.fr \\ ** LSG2M, École des Mines de Nancy \\ Parc de Saurupt, F-54042 Nancy cedex \\ benoit.appolaire@mines.inpl-nancy.fr
}

ABSTRACT. A general constitutive framework is proposed to incorporate linear and nonlinear mechanical behaviour laws into a standard phase field model. In the diffuse interface region where both phases coexist, two mixture rules for strain and stress are introduced, which are based on the Voigt/Taylor and Reuss/Sachs well-known homogenization schemes and compared to the commonly used mixture rules in phase field models. Finite element calculations have been performed considering an elastoplastic precipitate growing in an elastic matrix in order to investigate the plastic accommodation processes.

RÉSUMÉ. Nous proposons un cadre général permettant d'incorporer des lois de comportement mécanique non linéaires dans la méthode des champs de phases. Dans la région où les deux phases coexistent, deux techniques d'homogénéisation ont été appliquées, à savoir le modèle de Voigt/Taylor et le modèle de Reuss/Sachs, qui ont été comparées à une loi d'interpolation généralement utilisée dans les méthodes des champs de phases. Des calculs bidimensionnels par éléments finis ont été réalisés pour étudier la croissance d'un précipité élastoplastique dans une matrice élastique afin d'étudier les effets d'accommodation élasto-plastique.

KEYWORDS: phase field, elastoplasticity, homogenization.

MOTS-CLÉS : champs de phases, élastoplasticité, homogénéisation.

DOI:10.3166/EJCM.18.485-523 (c) 2009 Lavoisier, Paris 


\section{Introduction}

One observes in current literature a strong endeavour to develop microstructure evolution simulation schemes coupled with complex mechanical material behaviour ranging from heterogeneous elasticity to general elastoviscoplasticity. The main difficulty of such a task lies in the tight coupling between the complex interfaces evolutions and the fields, common to many free boundary problems. Hence, some attempts to achieve this goal have circumvented the difficulty by undertaking standard finite element calculations with prescribed interface kinetics, i.e. without the feedback of mechanics on phase transformation, e.g. (Ganghoffer et al., 1994; Barbe et al., 2008). If valuable insights have been obtained into internal stresses generated by evolving microstructures, the missing coupling prevents to use the predictions for materials undergoing phase transformations with complex changes in morphology and phases distribution. In parallel, the phase field approach has emerged as a powerful method for easily tackling the morphological evolutions involved in phase transformations. Phase field models have incorporated elasticity quite early (Onuki, 1989; Wang et al., 1993) and have succeeded in predicting some complex microstructure evolutions driven by the interplay of diffusion and elasticity, e.g. (Le bouar et al., 1998; Boussinot et al., 2009). It is only very recently that some phase field models have been enriched with nonlinear mechanical behaviour, extending the range of applications and materials which can be handled by the phase field approach (Guo et al., 2005; T. Uehara et al., 2007; Guo et al., 2008; Yamanaka et al., 2008; Zhou et al., 2008; Gaubert et al., 2008; Gaubert et al., 2009).

There are essentially two ways of introducing nonlinear mechanical constitutive equations into the standard phase field approach:

1) The material behaviour is described by a unified set of constitutive equations including material parameters that explicitly depend on the concentration or the phase variable. Each parameter is usually interpolated between the limit values known for each phase. This is the formulation adopted in the finite element simulations of CahnHilliard like equations coupled with viscoplasticity in (Ubachs et al., 2004; Ubachs et al., 2005) for tin-lead solders, also derived in (Forest, 2008; Forest, 2009). The same methodology is used in (Gaubert et al., 2008; Gaubert et al., 2009) to simulate the role of viscoplasticity on rafting of $\gamma^{\prime}$ precipitates in single crystal nickel base superalloys under load. For instance, when an elastic phase coexists with an elastic-plastic one, the plastic yield limit is interpolated between the real value in the plastic phase and a very high unreachable value in the elastic phase, e.g. (Cha et al., 2009).

2) One distinct set of constitutive equations is attributed to each individual phase $\alpha$ at any material point. Each phase at a material point then possesses its own stress/strain tensor $\sigma_{\alpha}, \varepsilon_{\alpha}$. The overall strain and stress quantities $\underset{\sim}{\sigma, \varepsilon} \underset{\sim}{ }$ at this material point must then be averaged or interpolated from the values attributed to each phases. This is particularly important for points inside the smooth interface zone. At this stage, several mixture rules are available to perform this averaging or interpolation. This approach makes possible to mix different types of constitutive equations for each phase, like hyperelastic nonlinear behaviour for one phase and conventional 
elasto-plastic model with internal variables for the other one. No correspondance of material parameters is needed between the phase behaviour laws. This is the approach proposed in (Steinbach et al., 2006) for incorporating elasticity in a multi-phase field model. For that purpose, the authors resort to a well-known homogeneous stress hypothesis taken from homogenization theory in the mechanics of heterogeneous materials (Nemat-Nasser et al., 1999; Qu et al., 2006). This approach has been applied to compute the effect of chemical induced strain on pearlite growth kinetics in (Steinbach et al., 2007). In the present work, we propose to generalize this procedure to nonlinear material behaviour and to other mixture rules also taken from homogenization theory. It must be emphazised that this procedure is very similar to what has already been proposed for handling diffusion in phase field models (Kim et al., 1999). Two concentration fields $c_{\alpha}$ and $c_{\beta}$ are indeed introduced, and the real concentration field is obtained by a mixture rule together with an internal constraint on the diffusion potentials, called quasi-equilibrium constraint in (Eiken et al., 2006). Introducing two concentration fields gives an additional degree of freedom for controlling the energy of the interface with respect to its thickness. If this possibility is not obvious when mechanics is introduced, adding a degree of freedom for describing the stresses/strains within a diffuse interface could be valuable to get rid of some spurious effects due to unrealistic interface thickness.

The objective of the present work is thus twofold:

1) To set a general framework that combines standard phase field approaches with a different complex mechanical behaviour for each phase. The approach will be shown to be amenable to practical simulations by presenting a simple finite element analysis of the growth of an elastic-plastic phase within an elastic matrix.

2) To compare the implication of the choice of specific mixture rules for these behaviour laws in the diffuse interface region on the predicted coherent phase diagram. Two interpolation rules taken from homogenization methods classically used in the mechanics of heterogeneous materials, will be evaluated and compared to the usual interpolation rule of standard phase field models. The comparison will be drawn for a simple microelasticity/phase field/diffusion problem for which an analytical solution is available.

The homogenization methods in the mechanics of heterogeneous materials have reached a high level of sophistication by providing bounds and estimations for the effective properties of elastic and nonlinear composites (Suquet, 1997; Jeulin et $a l ., 2001)$. They are based on the definition of a representative volume element (RVE) at each material point in which mean strain and stress can be defined for each phase. The basic assumption is that the local behaviour of phases inside the RVE can be represented by classical continuum mechanics. This is no longer the case when the RVE is a collection of atoms including different atom species, as it is usually the case in the volume element of phase field models. In particular the average relationships derived in continuum micromechanics are not valid for replacing a discrete set of atoms by a continuum with diffusion/mechanics effective properties. Discrete-continuum homogenization schemes exist in some cases for phase field models (Rodney et al., 2003) but 
remain limited in terms of physical situations. That is why no specific arrangement of phases will be considered inside the RVE for the theory proposed in this work. Instead, each relationship taken from micromechanical approaches will be adopted as a phenomenological ansatz in our model. Since there exists a large variety of such micromechanical mixture rules, it is worth evaluating some of them in the context of phase field/diffusion/mechanics models.

The numerical methods available to solve the coupled phase field/diffusion/mechanics field equations are those commonly used to solve partial differential equations. Hence, the finite volume scheme is adopted in (Appolaire et al., 2003; Appolaire et al., 2009) whereas a mixed finite difference-finite element scheme is used in (Nakajima et al., 2006). In (Gaubert et al., 2008), the Fourier method is used for periodic unit cell simulations. Finally, the simulations in (Ubachs et al., 2005) are carried out by means of a finite element method prevailing in the field of nonlinear mechanics. The simulations presented in this work are performed with the finite element model recently proposed in (Ammar et al., 2009a; Ammar et al., 2009c). Its setting is based on a variational formulation of the phase field equations in terms of generalized stresses as initially introduced by (Gurtin, 1996).

The paper is organized as follows. The balance and boundary conditions of a fully coupled phase field/diffusion/mechanical problem are given in Section2 The constitutive equations for chemical and mechanical processes are formulated by means of the expression of the free energy potential. A dissipation potential is then introduced for chemical and mechanical dissipative processes. A specific decomposition of these two potentials into chemical and mechanical contributions is given in Section 3 . Two mixture rules for strain and stress within the diffuse interface are analyzed in Section 4. which are based on the Voigt/Taylor and Reuss/Sachs well-known homogenization schemes. They are compared to the commonly used mixture rules in phase field models. Section 5 demonstrates that the choice of such an interpolation scheme can have serious consequences on the predicted coherent phase diagram. Consequently, calculating this phase diagram is used to rule out some unacceptable mixture rules. The last subsection illustrates the ability of our model to handle precipitation together with plasticity, in the simple case of one elastoplastic phase, including isotropic and kinematic hardening properties, growing into an elastic matrix.

For the sake of simplicity, the theory is expressed within the small perturbation framework (small strain), under isothermal conditions. The notations for all variables are summarized in Appendix A1. The notations used throughout this work are the following: zeroth, first, second and fourth-rank tensors are respectively denoted by $a, \underline{\boldsymbol{a}}, \underset{\sim}{\boldsymbol{a}}, \underset{\widetilde{a}}{\boldsymbol{a}}$. The simple and double contractions read . and :. The tensor product is denoted $\widetilde{b} y \otimes$. The nabla operator is denoted by $\nabla$. It is used extensively to compute the gradient or divergence of scalars, vectors and tensors. For example, $\boldsymbol{\nabla} . \boldsymbol{a}$ is the divergence of the second order tensor $\underset{\sim}{a}$. The gradient of the scalar $a$ is denoted by $\nabla a$. The time derivative of $a$ is $\dot{a}$. 


\section{Phase-field/diffusion/mechanical model}

\subsection{Principle of virtual power and balance equations}

The principle of virtual power has proved to be an efficient tool for deriving governing force balance equations and boundary conditions. Following this principle, the overall powers of internal, external and contact generalized forces, for all virtual order parameter $\phi^{\star}$ and virtual displacement $\underline{\boldsymbol{u}}^{\star}$ and for all subdomain $\mathcal{D}$ of body $V$, are assumed to admit power densities:

$$
\begin{gathered}
\mathcal{P}^{(i)}\left(\phi^{\star}, \underline{\boldsymbol{u}}^{\star}, V\right)=\int_{V} p^{(i)}\left(\phi^{\star}, \underline{\boldsymbol{u}}^{\star}\right) d v, \quad \mathcal{P}^{(e)}\left(\phi^{\star}, \underline{\boldsymbol{u}}^{\star}, V\right)=\int_{V} p^{(e)}\left(\phi^{\star}, \underline{\boldsymbol{u}}^{\star}\right) d v \\
\mathcal{P}^{(c)}\left(\phi^{\star}, \underline{\boldsymbol{u}}^{\star}, V\right)=\int_{\partial V} p^{(c)}\left(\phi^{\star}, \underline{\boldsymbol{u}}^{\star}\right) d s
\end{gathered}
$$

The power density of internal forces is taken as a general linear form, associated with generalized stresses $\{-\pi, \underline{\boldsymbol{\xi}}, \boldsymbol{\sigma}\}$ power-conjugates to $\left\{\phi^{\star}, \boldsymbol{\nabla} \phi^{\star}, \boldsymbol{\nabla} \underline{\boldsymbol{u}}^{\star}\right\}$ as:

$$
p^{(i)}\left(\phi^{\star}, \underline{\boldsymbol{u}}^{\star}\right)=\pi \phi^{\star}-\underline{\boldsymbol{\xi}} \cdot \boldsymbol{\nabla} \phi^{\star}-\underset{\sim}{\boldsymbol{\sigma}}: \boldsymbol{\nabla} \underline{\boldsymbol{u}}^{\star}
$$

where $\pi$ and $\boldsymbol{\xi}$ respectively are the scalar and vector microstresses, as introduced in (Gurtin, 1996) and $\underset{\sim}{\sigma}$ is the Cauchy stress tensor.

Similarly, the virtual power density of external generalized forces reads :

$$
p^{(e)}\left(\phi^{\star}, \underline{\boldsymbol{u}}^{\star}\right)=\gamma \phi^{\star}+\underline{\gamma} \cdot \nabla \phi^{\star}+\underline{\boldsymbol{f}} \cdot \underline{\boldsymbol{u}}^{\star}
$$

where $f$ is the volumic density of force and the external microforces are represented by the scalar $\gamma$ and the vector $\underline{\gamma}$ (Ammar et al., 2009a; Ammar et al., 2009b).

The contact generalized forces applied to the body are given by a surface density of microtraction $\zeta$ and a surface density of cohesion forces $\underline{t}$ for the purely mechanical part over the boundary. Then, the virtual power density of contact generalized forces is expressed as:

$$
p^{(c)}\left(\phi^{\star}, \underline{\boldsymbol{u}}^{\star}\right)=\zeta \phi^{\star}+\underline{\boldsymbol{t}} \cdot \underline{\boldsymbol{u}}^{\star}
$$

Assuming that no inertial microforces exist, the principle of virtual power requires that the virtual powers of externally and internally acting forces are balanced on any subdomain $\mathcal{D} \subset V$, for any choice of the virtual order parameter and displacement fields:

$$
\begin{aligned}
\forall \phi^{\star}, & \forall \underline{\boldsymbol{u}}^{\star}, \forall \mathcal{D} \subset V \\
& \mathcal{P}^{(i)}\left(\phi^{\star}, \underline{\boldsymbol{u}}^{\star}, \mathcal{D}\right)+\mathcal{P}^{(c)}\left(\phi^{\star}, \underline{\boldsymbol{u}}^{\star}, \mathcal{D}\right)+\mathcal{P}^{(e)}\left(\phi^{\star}, \underline{\boldsymbol{u}}^{\star}, \mathcal{D}\right)=0
\end{aligned}
$$




$$
\begin{aligned}
& \int_{\mathcal{D}}(\pi+\nabla \cdot \underline{\boldsymbol{\xi}}+\gamma-\nabla \cdot \underline{\boldsymbol{\gamma}}) \phi^{\star}+\left(\nabla \cdot \boldsymbol{\sigma}+\underline{\boldsymbol{f}}^{*} \cdot \underline{\boldsymbol{u}}^{\star} d v\right. \\
& +\int_{\partial \mathcal{D}}(\zeta-\underline{\boldsymbol{\xi}} \cdot \underline{\boldsymbol{n}}+\underline{\boldsymbol{\gamma}} \cdot \underline{\boldsymbol{n}}) \phi^{\star}+(\underline{\boldsymbol{t}}-\underline{\boldsymbol{\sigma}} \cdot \underline{\boldsymbol{n}}) \cdot \underline{\boldsymbol{u}}^{\star} d s=0
\end{aligned}
$$

The exploitation of the method of virtual power leads, on the one hand, to the balance equation associated with order parameter $\phi$ and boundary condition for the generalized microtraction vector:

$$
\boldsymbol{\nabla} \cdot(\underline{\boldsymbol{\xi}}-\underline{\boldsymbol{\gamma}})+\pi+\gamma=0 \quad \text { in } \quad V, \quad \text { and } \quad \zeta=(\underline{\boldsymbol{\xi}}-\underline{\boldsymbol{\gamma}}) \cdot \underline{\boldsymbol{n}} \quad \text { on } \quad \partial V \text { [7] }
$$

and, on the other hand, to the classical local static equilibrium and the associated boundary condition:

$$
\boldsymbol{\nabla} \cdot \boldsymbol{\sigma}+\underline{\boldsymbol{f}}=0 \quad \text { in } \quad V, \quad \text { and } \quad \underline{\boldsymbol{t}}=\boldsymbol{\sigma} \cdot \underline{\boldsymbol{n}} \quad \text { on } \quad \partial V
$$

The balance equation [7] is similar to Gurtin's microforce balance (Gurtin, 1996), with a slight extension represented by the presence of a possible prescribed volume density of vector external microforce $\underline{\gamma}$, which has been introduced for the sake of generality.

\subsection{Thermodynamical formulation}

The first principle of thermodynamics is formulated here in the absence of thermal and acceleration effects. The time variation of internal energy $\mathcal{E}$ is due to the power of external generalized forces, which is represented by volume and contact forces:

$$
\dot{\mathcal{E}}=\int_{V} \dot{e} d v=\mathcal{P}^{(e)}+\mathcal{P}^{(c)}
$$

where $e$ is the internal energy density.

Taking the principle of virtual power [5] into consideration, the local form of the energy principle then reads:

$$
\dot{e}=-\pi \dot{\phi}+\underline{\boldsymbol{\xi}} \cdot \nabla \dot{\phi}+\underset{\sim}{\sigma}: \underset{\sim}{\dot{\varepsilon}}
$$

where $\underset{\sim}{\varepsilon}$ is the total strain, which may be partitioned into the elastic strain ${\underset{\sim}{\varepsilon}}^{e}$, the eigenstrain ${\underset{\sim}{\star}}^{\star}$ due to phase transformation and the plastic strain $\underset{\sim}{\varepsilon^{p}}$ :

$$
\underset{\sim}{\varepsilon}={\underset{\sim}{\varepsilon}}^{e}+\underbrace{\star}_{\sim}+\underbrace{\varepsilon^{p}}_{\sim}
$$

According to the thermodynamics of irreversible processes, the second law states that the variation of entropy is always larger than or equal to the rate of entropy flux induced by diffusion: 


$$
T \dot{s}-\nabla \cdot(\mu \underline{\boldsymbol{J}}) \geq 0
$$

where $s$ is the entropy density, $\underline{\boldsymbol{J}}$ is the diffusion flux and $\mu$ is the chemical potential.

Introducing the free energy density $\dot{f}=\dot{e}-T \dot{s}$, and using the equation of mass balance $\dot{c}=-\nabla . \boldsymbol{J}$, the fundamental inequality containing first and second principles in the isothermal case is written as the Clausius-Duhem inequality:

$$
-\dot{f}-\pi \dot{\phi}+\underline{\boldsymbol{\xi}} \cdot \boldsymbol{\nabla} \dot{\phi}+\mu \dot{c}-\underline{\boldsymbol{J}} \cdot \boldsymbol{\nabla} \mu+\underset{\sim}{\boldsymbol{\sigma}}: \underset{\sim}{\dot{\boldsymbol{\varepsilon}}} \geq 0
$$

Assuming that the free energy density depends on the order parameter $\phi$ and its gradient, the concentration $c$, the elastic strain $\varepsilon^{e}$ and the set of internal variables $V_{k}$ associated to material hardening, the time derivation of $f$ with respect to its state variables is given by the chain rule as:

$$
\dot{f}\left(\phi, \nabla \phi, c, \underset{\sim}{\boldsymbol{\varepsilon}^{e}}, V_{k}\right)=\frac{\partial f}{\partial \phi} \dot{\phi}+\frac{\partial f}{\partial \boldsymbol{\nabla} \phi} \cdot \boldsymbol{\nabla} \dot{\phi}+\frac{\partial f}{\partial c} \dot{c}+\frac{\partial f}{\partial{\underset{\sim}{\varepsilon^{e}}}^{e}}:{\underset{\sim}{e}}^{e}+\frac{\partial f}{\partial V_{k}} \dot{V}_{k}
$$

Consequently, the Clausius-Duhem inequality becomes:

$$
\begin{aligned}
& -\left(\pi+\frac{\partial f}{\partial \phi}\right) \dot{\phi}+\left(\underline{\xi}-\frac{\partial f}{\partial \boldsymbol{\nabla} \phi}\right) \cdot \boldsymbol{\nabla} \dot{\phi}+\left(\mu-\frac{\partial f}{\partial c}\right) \dot{c}
\end{aligned}
$$

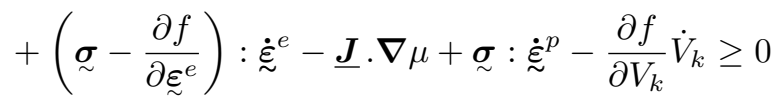

This inequality is linear in $\nabla \dot{\phi}, \dot{c}$ and $\underset{\sim}{\dot{\boldsymbol{\varepsilon}}^{e}}$. To ensure that the second law is satisfied in all conceivable processes and for any given thermodynamic variables $\left(\phi, \nabla \phi, c, \varepsilon^{e}, V_{k}\right)$, an analysis of the dissipation inequality leads to the following state laws (Coleman et al., 1963; Coleman et al., 1967):

$$
\underline{\boldsymbol{\xi}}=\frac{\partial f}{\partial \boldsymbol{\nabla} \phi} \quad, \quad \mu=\frac{\partial f}{\partial c} \quad \text { and } \quad \underset{\sim}{\boldsymbol{\sigma}}=\frac{\partial f}{\partial \underset{\sim}{\boldsymbol{\varepsilon}}}
$$

Similarly, we define the set of thermodynamic forces $A_{k}$ for each phase by derivation of the free energy density with respect to their associated internal variables:

$$
A_{k}=\frac{\partial f}{\partial V_{k}}
$$

Taking the above state laws into account, the Clausius-Duhem inequality [15] reduces to the residual dissipation:

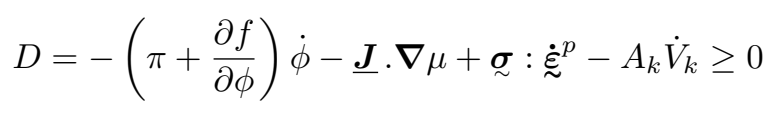


Three contributions appear in the above residual dissipation. The first is the phase field dissipation, associated with configuration changes of atoms and related to the evolution of the order parameter:

$$
D_{\phi}=-\pi_{\text {dis }} \dot{\phi} \quad \text { with } \quad \pi_{\text {dis }}=\pi+\frac{\partial f}{\partial \phi}
$$

where $\pi_{\text {dis }}$ is the chemical force associated with the dissipative processes (Gurtin, 1996).

The second contribution is the chemical dissipation due to the diffusion accommodation, associated with mass transport:

$$
D_{c}=-\underline{J} \cdot \nabla \mu
$$

and the last contribution is called mechanical dissipation due to the evolution of the internal variables, which is represented by the products of the thermodynamic force variables with their respective rates of internal variable:

$$
D_{u}=\underset{\sim}{\sigma}:{\underset{\sim}{\dot{\varepsilon}}}^{p}-A_{k} \dot{V}_{k}
$$

An efficient way of defining the complementary laws related to the dissipative processes and ensuring the positivity of the dissipation for any thermodynamic processes is to assume the existence of a dissipation potential $\Omega\left(\pi_{\mathrm{dis}}, \nabla \mu, \boldsymbol{\sigma}, A_{k}\right)$, which is a convex function of its arguments:

$$
\begin{aligned}
& \dot{\phi}=-\frac{\partial \Omega}{\partial \pi_{\mathrm{dis}}} \\
& \underline{\boldsymbol{J}}=-\frac{\partial \Omega}{\partial \boldsymbol{\nabla} \mu} \\
& \dot{V}_{k}=-\frac{\partial \Omega}{\partial A_{k}} \quad, \quad \dot{\sim}^{p}=\frac{\partial \Omega}{\partial \boldsymbol{\sigma}}
\end{aligned}
$$

These equations represent the evolution law for the order parameter [22], the diffusion flux [23] as well as the evolution laws for the internal variables [24].

\section{Free energy and dissipation potential}

\subsection{Partition of free energy and dissipation potential}

The proposed model is formulated within the framework of generalized standard materials (Germain et al., 1983), where the complete elastoplastic behaviour can be 
described by the knowledge of two potentials. These potentials are namely the thermodynamic potential, which is the total free energy, for the reversible aspects and the dissipative potential related to the dissipative processes.

The total free energy is postulated to have the form of a Ginzburg-Landau free energy functional accounting for interfaces through the square of the order parameter gradient. The total free energy $F$ of the body is then defined by the integral over the volume $V$ of a free energy density $f$, which can be split into a chemical free energy density $f_{\text {ch }}$, a coherent mechanical energy density $f_{u}$, and the square of the order parameter gradient:

$$
\begin{aligned}
F\left(\phi, \nabla \phi, c,{\underset{\sim}{e}}^{e}, V_{k}\right) & =\int_{V} f\left(\phi, \nabla \phi, c,{\underset{\sim}{\varepsilon}}^{e}, V_{k}\right) d v \\
& =\int_{V}\left[f_{\mathrm{ch}}(\phi, c)+f_{u}\left(\phi, c, \underset{\sim}{\varepsilon}, V_{k}\right)+\frac{\alpha}{2}|\nabla \phi|^{2}\right] d v[25]
\end{aligned}
$$

The irreversible behaviour is described by the introduction of a dissipation potential, which can be split into three parts related to the three contributions in the residual dissipation in [18]: the phase field part $\Omega_{\phi}\left(\phi, c, \pi_{\mathrm{dis}}\right)$, the chemical part $\Omega_{c}(\phi, c, \nabla \mu)$ and the mechanical dissipation potential $\Omega_{u}\left(\phi, c, \boldsymbol{\sigma}, A_{k}\right)$ :

$$
\Omega\left(\pi_{\mathrm{dis}}, \nabla \mu, \phi, c, \boldsymbol{\sigma}, A_{k}\right)=\Omega_{\phi}\left(\pi_{\mathrm{dis}}\right)+\Omega_{c}(\nabla \mu)+\Omega_{u}\left(\phi, c, \boldsymbol{\sigma}, A_{k}\right)
$$

\subsection{Chemical contribution}

The chemical free energy density $f_{\text {ch }}$ of the binary alloy is a function of the order parameter $\phi$ and of the concentration field $c$. In order to guarantee the coexistence of both phases $\alpha$ and $\beta$ discriminated by $\phi, f_{\text {ch }}$ must be non-convex with respect to $\phi$. Following (Kim et al., 1998), $f_{\mathrm{ch}}$ is built with the free energy densities of the two phases $f_{\alpha}$ and $f_{\beta}$ as follows:

$$
f_{\mathrm{ch}}(\phi, c)=h(\phi) f_{\alpha}(c)+[1-h(\phi)] f_{\beta}(c)+W g(\phi)
$$

Here, $h(\phi)$ is chosen as $h(\phi)=\phi^{2}(3-2 \phi)$, and $g(\phi)=\phi^{2}(1-\phi)^{2}$ is the double well potential accounting for the free energy penalty of the interface. The height $W$ of the potential barrier is related to the interfacial energy $\sigma$ and the interfacial thickness $\delta$ as $W=6 \Lambda \sigma / \delta$. Assuming that the interface region ranges from $\theta$ to $1-\theta$, then $\Lambda=\ln [(1-\theta) / \theta]$. In the present work $\theta=0.05$ (Kim et al., 1998; Ammar et al., 2009a).

The densities $f_{\alpha}$ and $f_{\beta}$ are chosen to be quadratic functions of the concentration only:

$$
f_{\alpha}(c)=\frac{k_{\alpha}}{2}\left(c-a_{\alpha}\right)^{2} \quad \text { and } \quad f_{\beta}(c)=\frac{k_{\beta}}{2}\left(c-a_{\beta}\right)^{2}
$$


where $a_{\alpha}$ and $a_{\beta}$ are the unstressed equilibrium concentrations of both phases which correspond respectively to the minima of $f_{\alpha}$ and $f_{\beta}$ in the present model. $k_{\alpha}$ and $k_{\beta}$ are the curvatures of the free energies.

Moreover, the positiveness of the intrinsic dissipation is ensured by the choice of a convex dissipation potential of its arguments. Consequently, the chemical and phase field dissipation potentials read:

$$
\Omega_{\phi}\left(\pi_{\mathrm{dis}}\right)=\frac{1}{2}(1 / \beta) \pi_{\mathrm{dis}}^{2} \quad \text { and } \quad \Omega_{c}(\nabla \mu)=\frac{1}{2} L(\phi) \nabla \mu \cdot \nabla \mu
$$

where $\pi_{\text {dis }}$ is given by Eq. [19], $\beta$ is inversely proportional to the interface mobility and $L(\phi)$ is the Onsager coefficient, related to the chemical diffusivities $D_{\alpha}$ and $D_{\beta}$ in both phases by means of the interpolation function $h(\phi)$ as:

$$
L(\phi)=h(\phi) D_{\alpha} / k_{\alpha}+(1-h(\phi)) D_{\beta} / k_{\beta}
$$

Once the particular forms of free energy [25] ] and dissipation potential [29] are known, the state laws [16] and the complementary evolutions Eqs. [23 24] for the phase field and chemical contributions can be derived as:

$$
\begin{aligned}
& \underline{\boldsymbol{\xi}}=\alpha \boldsymbol{\nabla} \phi, \mu=\frac{\partial f_{\mathrm{ch}}}{\partial c}+\frac{\partial f_{u}}{\partial c} \\
& \dot{\phi}=-(1 / \beta) \pi_{\mathrm{dis}}=-(1 / \beta)\left(\pi+\frac{\partial f_{\mathrm{ch}}}{\partial \phi}+\frac{\partial f_{u}}{\partial \phi}\right) \quad, \underline{\boldsymbol{J}}=-L(\phi) \nabla \mu
\end{aligned}
$$

Substituting the previous equations into the balance equations for generalized stresses and mass concentration, the Ginzburg-Landau and usual diffusion equations are retrieved, which represent respectively the evolution equations for order parameter and concentration:

$$
\begin{aligned}
& \boldsymbol{\nabla} \underline{\boldsymbol{\xi}}+\pi=-\beta \dot{\phi}+\alpha \Delta \phi-\frac{\partial f_{\mathrm{ch}}}{\partial \phi}-\frac{\partial f_{u}}{\partial \phi}=0 \\
& \dot{c}=-\nabla \cdot(-L(\phi) \nabla \mu)=-\nabla \cdot\left[-L(\phi)\left(\nabla \frac{\partial f_{\mathrm{ch}}}{\partial c}+\nabla \frac{\partial f_{u}}{\partial c}\right)\right]
\end{aligned}
$$

\subsection{Mechanical contribution}

The second contribution to the free energy density is due to mechanical effects. Assuming that elastic behaviour and hardening are uncoupled, the mechanical part of the free energy density $f_{u}$ is decomposed into a coherent elastic energy density $f_{e}$ and a plastic part $f_{p}$ as:

$$
f_{u}\left(\phi, c, \varepsilon, V_{k}\right)=f_{e}(\phi, c, \varepsilon)+f_{p}\left(\phi, c, V_{k}\right)
$$


Moreover, the irreversible mechanical behaviour, related to the dissipative processes, is obtained by a plastic dissipation potential $\Omega_{u}\left(\phi, c, \boldsymbol{\sigma}, A_{k}\right)$. It is assumed to be a function of order parameter, concentration, Cauchy stress tensor as well as the set of thermodynamic force associated variables $A_{k}$ in order to describe the hardening state in each phase. The specific form of $f_{u}\left(\phi, c, \varepsilon, V_{k}\right)$ and $\Omega_{u}\left(\phi, c, \sigma, A_{k}\right)$ will be detailed in the next sections.

\section{Phase field approach and homogenization methods}

\subsection{Multiphase approach}

In the region where both phases coexist, we propose to use well-known results of homogenization theory to interpolate the local behaviour. The homogenization procedure in the mechanics of heterogeneous materials consists in replacing an heterogeneous medium by an equivalent homogeneous one, which is defined by an effective constitutive law relating the macroscopic variables, namely macroscopic stress $\sigma$ and strain $\underset{\sim}{\varepsilon}$ tensors, which are obtained by averaging the corresponding non-uniform local stress and strain in each phase. In the case of a two-phase materials:

$$
\underset{\sim}{\boldsymbol{\varepsilon}}=\frac{1}{\mathcal{V}} \sum_{k=\alpha, \beta} \int_{\mathcal{V}_{k}}{\underset{\beth}{\varepsilon}}_{k} d v \quad \text { and } \quad \underset{\sim}{\boldsymbol{\sigma}}=\frac{1}{\mathcal{V}} \sum_{k=\alpha, \beta} \int_{\mathcal{V}_{k}} \boldsymbol{\sigma}_{k} d v
$$

where $\mathcal{V}=\mathcal{V}_{\alpha} \cup \mathcal{V}_{\beta}$ is the underlying material representative volume element.

Following a naive representation depicted in Figure 1 each material point, i.e. $\mathcal{V}$, within a diffuse interface can be seen as a local mixture of the two abutting phases $\alpha$ and $\beta$ with proportions fixing $\mathcal{V}_{\alpha}$ and $\mathcal{V}_{\beta}$ given by complementary functions of $\phi$. It must be emphasized that this representation involves the presence of fields $\Psi_{\alpha}$ and $\Psi_{\beta}$ in phases $\beta$ and $\alpha$ respectively, which has no incidence on the bulk of those phases.

For illustrating the way to apply homogenization schemes in a diffuse interface, the case of two elastoplastic materials with hardening can be considered. The strain and stress at each material point are defined by the following mixture laws which would proceed from space averaging in a conventional homogenization problem, but which must be seen as arbitrary interpolations in the present case:

$$
\underset{\sim}{\boldsymbol{\varepsilon}}=\chi \underset{\sim}{\boldsymbol{\varepsilon}_{\alpha}}+(1-\chi) \boldsymbol{\varepsilon}_{\beta} \quad \text { and } \quad \underset{\sim}{\boldsymbol{\sigma}}=\chi \underset{\sim}{\boldsymbol{\sigma}}+(1-\chi) \underset{\sim}{\boldsymbol{\sigma}_{\beta}}
$$

where $\boldsymbol{\varepsilon}_{\alpha}, \boldsymbol{\varepsilon}_{\beta}$ are the local strains and $\boldsymbol{\sigma}_{\alpha}, \boldsymbol{\sigma}_{\beta}$ are the local stresses in $\alpha$ and $\beta$ phases respectively and $\chi(\underline{\boldsymbol{x}}, t)$ is a shape function which must verify:

$$
\left\{\begin{array}{l}
\chi(\underline{\boldsymbol{x}}, t)=0 \text { if } \underline{\boldsymbol{x}} \in \beta \\
\chi(\underline{\boldsymbol{x}}, t)=1 \text { if } \underline{\boldsymbol{x}} \in \alpha
\end{array}\right.
$$




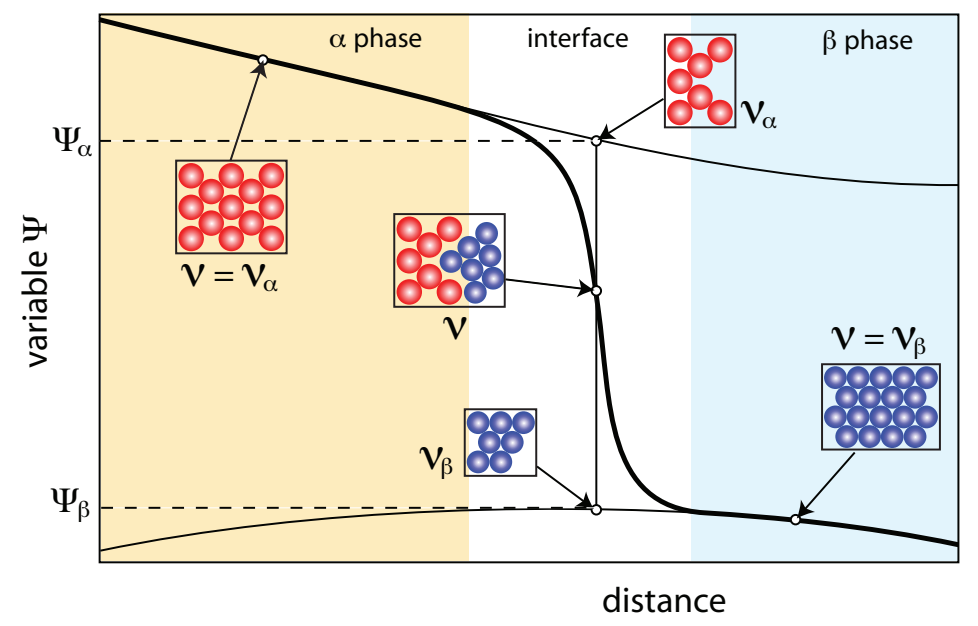

Figure 1. Schematic illustration of the underlying material representative volume element $\mathcal{V}$ at each material point of a diffuse interface: the real effective variable $\Psi$ appears with a thick line, whereas the variables attached to each phase $\Psi_{\alpha}$ and $\Psi_{\beta}$ are with thin lines

In this work, the simplest choice has been done for this shape function:

$$
\chi(\underline{\boldsymbol{x}}, t)=\phi(\underline{\boldsymbol{x}}, t)
$$

The partition hypothesis, already used for the effective total strain tensor in [11], requires, in a similar way, a decomposition of the total strain in each phase into elastic, eigen and plastic parts:

$$
\varepsilon_{\sim}=\varepsilon_{\sim \alpha}^{e}+{\underset{\sim}{\varepsilon_{\alpha}}}^{\star}+{\underset{\sim}{\varepsilon}}_{\alpha}^{p} \text { and }{\underset{\sim}{\varepsilon} \beta}_{\beta}{\underset{\sim}{\beta}}_{\beta}^{e}+\varepsilon_{\beta}^{\star}+\varepsilon_{\sim}^{p}
$$

where each point may depend on the local concentration $c$, but not in order parameter $\phi$.

In the proposed model, the elastoplastic and phase field behaviours of each phase are treated independently and the effective behaviour is obtained using homogenization relation [37]. It is assumed that the mechanical state of $\alpha$ and $\beta$ phases at a given time are completely described by a finite number of local state variables, defined at each point as:

$$
\left({\underset{\varepsilon}{k}}_{k},{\underset{\varepsilon}{p}}_{k}^{p}, V_{k}\right) \quad \text { where } \quad V_{k}=\left(r_{k},{\underset{\sim}{\alpha}}_{k}\right) \text { and } k=\{\alpha, \beta\}
$$

The set of internal variables $V_{k}$, of scalar or tensorial nature, represents the state of hardening of phase $k$ : for instance, a scalar isotropic hardening variable $r_{k}$, and a tensorial kinematic hardening variable $\underset{\sim}{\boldsymbol{\alpha}}$. 
The reversible mechanical behaviour of each individual phase is governed by a convex mechanical free energy, which can decomposed, using Eq. [35], into local elastic and plastic energy densities. According to the homogenization theory, the effective elastic and plastic free energy densities are given by the rule of mixtures as follows:

$$
f_{e}(\phi, c, \underset{\sim}{\varepsilon})=\phi f_{e \alpha}\left(c, \varepsilon_{\alpha}^{e}\right)+(1-\phi) f_{e \beta}\left(c, \varepsilon_{\beta}^{e}\right)
$$

and

$$
f_{p}\left(\phi, c, V_{k}\right)=\phi f_{p \alpha}\left(c, \boldsymbol{\alpha}_{\alpha}\right)+(1-\phi) f_{p \beta}\left(c, \boldsymbol{\alpha}_{\beta}\right)
$$

Similarly, we define a set of thermodynamic forces $A_{k}=\left(R_{k}, \underset{\sim}{\boldsymbol{X}_{k}}\right)$ associated with the internal variables $V_{k}=\left(r_{k}, \boldsymbol{\alpha}_{k}\right)$ for each phase, represented by the scalar isotropic hardening $R_{k}$ and the tensor of kinematic hardening $\underset{\sim}{\boldsymbol{X}}$.

Consequently, the Cauchy stress tensor and the associated thermodynamic force variables for both phases are deduced from Eqs. [16]17] as:

$$
\begin{array}{ll}
\boldsymbol{\sigma}_{\alpha}=\frac{\partial f_{e \alpha}}{\partial \boldsymbol{\varepsilon}_{\alpha}} \quad, \quad{\underset{\sim}{\beta}}_{\beta}=\frac{\partial f_{e \beta}}{\partial \boldsymbol{\varepsilon}_{\beta}} \\
R_{\alpha}=\frac{\partial f_{p \alpha}}{\partial r_{\alpha}} \quad, \quad R_{\beta}=\frac{\partial f_{p \beta}}{\partial r_{\beta}} \\
\underset{\sim}{\boldsymbol{X}}=\frac{\partial f_{p \alpha}}{\partial \boldsymbol{\alpha}_{\alpha}} \quad, \quad \underset{\sim}{\boldsymbol{X}}=\frac{\partial f_{p \beta}}{\partial \boldsymbol{\alpha}_{\beta}}
\end{array}
$$

In order to describe the irreversible part of mechanical behaviour in each phase, we define the local mechanical dissipation potentials $\Omega_{u \alpha}\left(c, \boldsymbol{\sigma}_{\alpha}, A_{\alpha}\right)$ and $\Omega_{u \beta}\left(c, \boldsymbol{\sigma}_{\beta}, A_{\beta}\right)$ for $\alpha$ and $\beta$ phases respectively, which are convex functions of their arguments. Consequently, the mechanical potential for effective material is defined with respect to the mechanical potentials in both phases by means of the shape function $\phi$ as:

$$
\Omega_{u}\left(\phi, c, \underset{\sim}{\boldsymbol{\sigma}}, A_{k}\right)=\phi \Omega_{u \alpha}\left(c, \boldsymbol{\sigma}_{\alpha}, A_{\alpha}\right)+(1-\phi) \Omega_{u \beta}\left(c, \boldsymbol{\sigma}_{\beta}, A_{\beta}\right)
$$

Using Eqs. [24], the complementary evolution laws of the internal variables in the two phases are derived from the above potential as follows:

$$
\begin{aligned}
& \dot{\sim}_{\alpha}^{p}=\frac{\partial \Omega_{u \alpha}}{\partial \boldsymbol{\sigma}_{\alpha}} \quad, \quad \dot{\boldsymbol{\varepsilon}}_{\beta}^{p}=\frac{\partial \Omega_{u \beta}}{\partial \boldsymbol{\sigma}_{\beta}} \\
& \dot{r}_{\alpha}=-\frac{\partial \Omega_{u \alpha}}{\partial R_{\alpha}} \quad, \quad \dot{r}_{\beta}=-\frac{\partial \Omega_{u \beta}}{\partial R_{\beta}} \\
& {\underset{\sim}{\alpha}}_{\alpha}=-\frac{\partial \Omega_{u \alpha}}{\partial \underset{\sim}{\boldsymbol{X}}} \quad, \quad \dot{\sim}_{\beta}=-\frac{\partial \Omega_{u \beta}}{\partial \underset{\sim}{\boldsymbol{X}_{\beta}}}
\end{aligned}
$$




\subsection{Voigt/Taylor model}

Voigt's model is also referred to as the isostrain model. Its basic assumptions are that the strain field is uniform among the phases (Nemat-Nasser et al., 1999; Qu et al., 2006). Using Voigt's model, we assume a uniform total strain at any point in the diffuse interface between elastoplastically inhomogeneous phases. The effective stress is expressed in terms of the local stress average with respect to both phases weighted by the volume fractions:

$$
\underset{\sim}{\boldsymbol{\sigma}}=\phi{\underset{\sim}{\boldsymbol{\sigma}}}_{\alpha}+(1-\phi){\underset{\sim}{\boldsymbol{\sigma}}}_{\beta} \quad, \quad \underset{\sim}{\boldsymbol{\varepsilon}}=\boldsymbol{\varepsilon}_{\alpha}=\boldsymbol{\varepsilon}_{\beta}
$$

The stresses of both phases $\boldsymbol{\sigma}_{\alpha}$ and $\boldsymbol{\sigma}_{\beta}$ are given by Hooke's law for each phase:

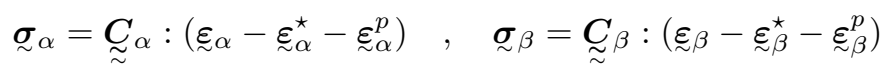

where $\underset{\sim}{\boldsymbol{C}_{\alpha}}$ and $\underset{\sim}{\boldsymbol{C}} \beta$ are respectively the tensor of elasticity moduli in $\alpha$ and $\beta$ phases.

The stress at any point in the interface is computed from the average of the above local stresses as follows:

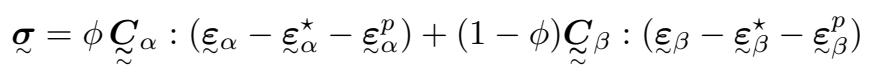

From the above relation, it follows that the strain-stress relationship in the homogeneous effective medium obeys Hooke's law with the following equation:

$$
\underset{\sim}{\sigma}=\underset{\sim}{\boldsymbol{C}} \text { eff }:\left(\underset{\sim}{\varepsilon}-{\underset{\sim}{\varepsilon}}^{p}-{\underset{\sim}{\star}}^{\star}\right)
$$

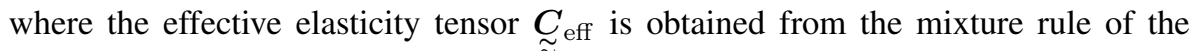
elasticity matrix for both phases:

$$
\underset{\sim}{\boldsymbol{C}} \text { eff }=\phi \underset{\sim}{\boldsymbol{C}_{\alpha}}+(1-\phi) \underset{\sim}{\boldsymbol{C}_{\beta}}
$$

and the effective eigenstrain $\varepsilon_{\sim}^{\star}$ and plastic strain ${\underset{\sim}{p}}^{p}$ vary continuously between their respective values in the bulk phases as follows:

$$
\begin{aligned}
& {\underset{\sim}{\boldsymbol{\varepsilon}}}^{\star}=\underset{\sim}{\boldsymbol{C}} \mathrm{eff}^{-1}:\left(\phi \underset{\sim}{\boldsymbol{C}} \alpha:{\underset{\sim}{\boldsymbol{\varepsilon}}}_{\alpha}^{\star}+(1-\phi) \underset{\sim}{\boldsymbol{C}}{ }_{\beta}:{\underset{\sim}{\boldsymbol{\varepsilon}}}_{\beta}^{\star}\right) \\
& {\underset{\sim}{\boldsymbol{\varepsilon}}}^{p}=\underset{\approx}{\boldsymbol{C}} \mathrm{eff}^{-1}:\left(\phi \underset{\sim}{\boldsymbol{C}} \alpha: \underset{\sim \alpha}{\boldsymbol{\varepsilon}_{\alpha}^{p}}+(1-\phi) \underset{\approx}{\boldsymbol{C}} \beta:{\underset{\sim}{\boldsymbol{\varepsilon}} p}_{\beta}^{p}\right)
\end{aligned}
$$

In the case of nonhomogeneous elasticity, it must be noted that $\varepsilon^{\star}$ and ${\underset{\sim}{p}}^{p}$ are not the average of their respective values for each phase.

The local energy stored in the effective homogeneous elastic material is expressed in terms of the average value of the local elastic energy with respect to both phases weighted by their volume fractions:

$$
\begin{aligned}
f_{e} & =\frac{1}{2}\left({\underset{\sim}{\varepsilon}-\sim^{\star}}_{\varepsilon^{\star}}^{\varepsilon^{p}}\right):{\underset{\sim}{C}}_{\text {eff }}:\left(\sim_{\sim}^{\varepsilon}-{\underset{\sim}{\varepsilon}}^{\star}-{\underset{\sim}{\varepsilon}}^{p}\right) \\
& =\phi f_{e \alpha}+(1-\phi) f_{e \beta}
\end{aligned}
$$


where the elastic energy densities of $\alpha$ and $\beta$ phases can be expressed as:

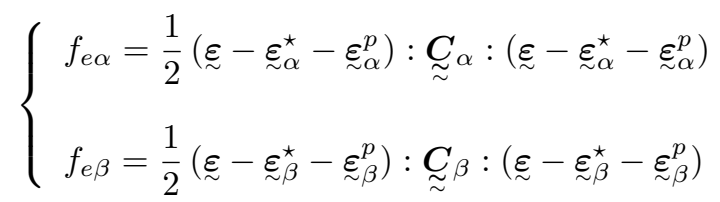

\subsection{Reuss/Sachs model}

The Reuss/Sachs scheme assumes homogeneity of stress among the phases. The effective strain is obtained by averaging the corresponding strains in each phase:

$$
\underset{\sim}{\boldsymbol{\sigma}}={\underset{\sim}{\alpha}}_{\alpha}=\boldsymbol{\sigma}_{\beta} \quad, \quad \underset{\sim}{\boldsymbol{\varepsilon}}=\phi \underset{\sim}{\boldsymbol{\varepsilon}} \alpha+(1-\phi) \underset{\sim}{\boldsymbol{\varepsilon}_{\beta}}
$$

Taking Hooke's law into account for each phase, the local total strain in each phase is expressed:

$$
\underset{\sim}{\boldsymbol{\varepsilon}}=\underset{\sim}{\boldsymbol{S}}{ }_{\alpha}: \boldsymbol{\sigma}_{\alpha}+\boldsymbol{\sim}_{\alpha}^{\star}+{\underset{\sim}{\boldsymbol{\varepsilon}}}_{\alpha}^{p} \quad, \quad{\underset{\sim}{\boldsymbol{\varepsilon}}}_{\beta}=\underset{\sim}{\boldsymbol{S}} \boldsymbol{\sim}_{\beta}: \boldsymbol{\sigma}_{\beta}+\boldsymbol{\sim}_{\beta}^{\star}+\boldsymbol{\sim}_{\beta}^{p}
$$

where $\underset{\sim}{\boldsymbol{S}_{\alpha}}$ and $\underset{\sim}{\boldsymbol{S}} \beta$ are the tensors of elastic compliance of each phase.

Taking $[58]_{2}$ into account, the effective strain is found as:

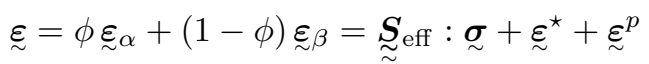

where the expression of the effective eigenstrain $\varepsilon_{\sim}^{\star}$, the effective plastic strain $\varepsilon_{\sim}^{p}$ and the effective compliance matrix $\underset{\sim}{\boldsymbol{S}}$ eff are defined as the average of the local properties of each phase:

$$
\begin{aligned}
& \varepsilon_{\sim}^{\star}=\phi{\underset{\sim}{\alpha}}_{\alpha}^{\star}+(1-\phi) \varepsilon_{\beta}^{\star} \quad, \quad{\underset{\sim}{\sim}}^{p}=\phi \varepsilon_{\alpha}^{p}+(1-\phi) \varepsilon_{\beta}^{p} \\
& \underset{\sim}{\boldsymbol{S}} \text { eff }=\phi \underset{\sim}{\boldsymbol{\sim}_{\alpha}}+(1-\phi) \underset{\sim}{\boldsymbol{S}} \beta
\end{aligned}
$$

Let us consider a homogeneous material with elastic stiffness $\underset{\approx}{\boldsymbol{S}}-1$. For a given effective elastic strain, the stress is given by Hooke's law as:

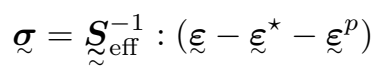

Similar to Voigt's model, the elastic energy density of the material is constructed as follows:



$$
\begin{aligned}
& =\phi f_{e \alpha}+(1-\phi) f_{e \beta}
\end{aligned}
$$

where $f_{e \alpha}$ and $f_{e \beta}$ represent elastic energy densities of $\alpha$ and $\beta$ phases respectively. They are still given by with [57] with $\underset{\sim}{\boldsymbol{C}} \alpha, \beta=\underset{\sim}{\boldsymbol{S}}-1, \beta$. 


\subsection{Comparison with existing interpolation schemes}

The interface behaviours proposed above can be compared to the most commonly used in phase field models, as popularized by Khachaturyan and coworkers, e.g. (Khachaturyan, 1983). According to these works, mixture rules are adopted respectively for eigenstrain and elasticity moduli tensor:

$$
\underline{\sim}^{\star}=\phi{\underset{\sim}{\varepsilon_{\alpha}}}^{\star}+(1-\phi){\underset{\sim}{\varepsilon_{\beta}}}^{\star} \quad, \quad \underset{\sim}{\boldsymbol{C}} \text { eff }=\phi \underset{\sim}{\boldsymbol{C}} \alpha+(1-\phi) \underset{\sim}{\boldsymbol{C}} \beta
$$

Hooke's law relates the strain tensor to the stress tensor by the following expression:

$$
\begin{aligned}
& \underset{\sim}{\sigma}={\underset{\sim}{(} \text { eff }}:\left(\underset{\sim}{\varepsilon}-{\underset{\sim}{*}}^{\star}\right)
\end{aligned}
$$



Contrary to homogenization schemes, it is clear that the elastic energy of the effective homogeneous material is no longer the average of energy densities of both phases. It is indeed not possible to distinguish an explicit form for the elastic energy densities in each phase. The elastic energy is then postulated as:

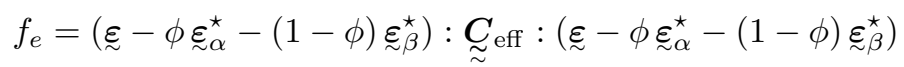

As a result, it appears that Eq. $[64]_{1}$ corresponds to Reuss' approach whereas Eq. $\llbracket 64]_{2}$ is taken from Voigt's model. The standard phase field microelasticity approach therefore combines two homogenization schemes so that it is not possible to identify consistent definitions for strain/stress components for each phase. Moreover, the study of the plastic accommodation effects in the phase field interface, using this model, requires the definition of additional conditions. They relate the effective plastic activity to the local plastic behaviour of the two phases, such as the linear mixture model of the material parameters (Ubachs et al., 2005). An interpolation scheme of the plastic parameters by means of tanh function has been proposed in (Gaubert et al., 2008).

\subsection{Expression of the phase field-elastic coupling terms}

In the phase field approach to coherent phase transformations, there are in general three contributions to the interfacial energy: the first one coming from the double well function $g(\phi)$, the second one coming from the variation in concentration within the interface and the last one is due to mechanically stored energy. After substituting the free energy density [57] into [33], the time evolution of the phase field at thermodynamic equilibrium $\left(\phi=\phi_{\text {eq }}\right)$ become:

$$
\begin{aligned}
\alpha \frac{d^{2} \phi}{d x^{2}} & =\frac{\partial f_{0}}{\partial \phi}+\frac{\partial f_{e}}{\partial \phi} \\
\alpha \frac{d^{2} \phi}{d x^{2}} & =\frac{\partial f_{0}}{\partial \phi}+\frac{\partial \boldsymbol{\varepsilon}^{e}}{\partial \phi}: \underset{\sim}{\boldsymbol{C}_{\mathrm{eff}}}: \boldsymbol{\sim}^{e}+\frac{1}{2}{\underset{\sim}{\boldsymbol{\tau}}}^{e}: \frac{\partial \underset{\widetilde{\partial} \phi}{\boldsymbol{e}} \mathrm{eff}}{\varepsilon^{e}}
\end{aligned}
$$


The order parameter evolution for coherent transformation is extended to include the variation of the elastic free energy as an elastic driving force for the phase transformation process. It characterizes the effect of mechanically stored energy on the phase field and its evolution. The derivation of the elastic strain and the effective elasticity tensor with respect to the order parameter for the various interpolation schemes are summarised below.

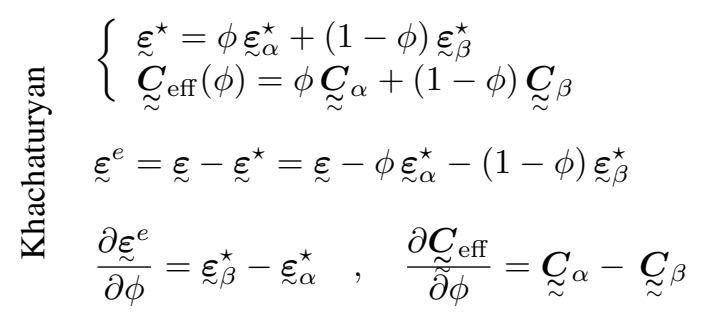

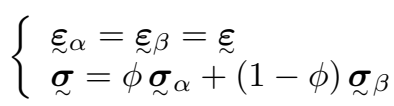



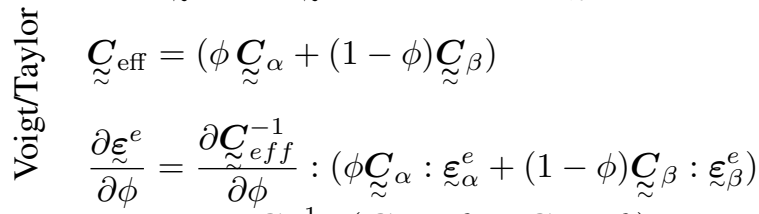

$$
\begin{aligned}
& +\underset{\sim}{\boldsymbol{C}} \text { eff }:\left(\underset{\sim}{\boldsymbol{C}} \alpha:{\underset{\sim}{\alpha}}_{\alpha}^{e}-\underset{\approx}{\boldsymbol{C}_{\beta}}:{\underset{\sim}{\boldsymbol{\varepsilon}}}_{\beta}^{e}\right)
\end{aligned}
$$



$$
\begin{aligned}
& \left\{\begin{array}{l}
\boldsymbol{\varepsilon}=\phi \varepsilon_{\alpha}+(1-\phi) \boldsymbol{\varepsilon}_{\beta} \\
{\underset{\sim}{\alpha}}_{\alpha}={\underset{\sim}{\sigma}}_{\beta}=\boldsymbol{\sigma}
\end{array}\right.
\end{aligned}
$$

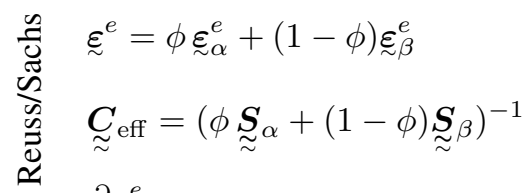






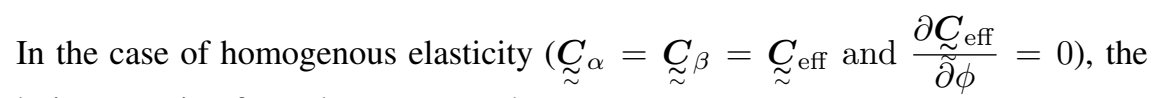
evolution equation for order parameter becomes:

$$
\alpha \frac{d^{2} \phi}{d x^{2}}=\frac{\partial f_{0}}{\partial \phi}+\frac{\partial \mathcal{\varepsilon}^{e}}{\partial \phi}: \underset{\sim}{\boldsymbol{C f f f}}:{\underset{\sim}{\varepsilon}}^{e}
$$

where

$$
\begin{cases}\frac{\partial \varepsilon_{\sim}^{e}}{\partial \phi}=0 & \text { for Reuss/Sachs scheme } \\ \frac{\partial \varepsilon_{\sim}^{e}}{\partial \phi}=\varepsilon_{\sim}^{\star}-\varepsilon_{\alpha}^{\star} & \text { for Voigt/Taylor and Khachaturyan schemes }\end{cases}
$$

Using Reuss' model, we clearly show that there is no mechanical contribution on the phase field equation in the case of homogenous elasticity. It must be noted that this equation is different from equation [24] presented in (Steinbach et al., 2006) for the same scheme. Moreover, the same mechanical contribution $\left(\frac{\partial \varepsilon_{\mathcal{\varepsilon}}^{e}}{\partial \phi}={\underset{\sim}{\varepsilon}}_{\beta}^{\star}-\varepsilon_{\alpha}^{\star}\right)$ on the phase field equation is shown for Voigt/Taylor and Khachaturyan schemes.

\section{Applications}

\subsection{Implementation in a finite element code}

The set of partial differential equations, which are the local static equilibrium, the balance of generalized stresses and the balance of mass, is solved using a finite element method to discretize space and a finite difference method to discretize time. The implementation of the proposed model is made in the finite element code ZeBuLon (Foerch et al., 1997), where order parameter, concentration and displacement are taken as nodal degrees of freedom $\left(\phi, c, u_{i}\right)$. Regarding time discretization, the differential equations are integrated at each Gauss point in an incremental procedures using a Euler implicit scheme $(\theta$-method). Variational formulation of the phase field equation and the local static equilibrium are derived from the principle of virtual power Eq. [5] (Ammar et al., 2009a; Ammar et al., 2009c):

$$
\begin{aligned}
& \Im\left(\phi^{\star}\right)=\int_{V}\left(\pi \phi^{\star}-\underline{\boldsymbol{\xi}} \cdot \nabla \phi^{\star}\right) d v+\int_{\partial V} \zeta \phi^{\star} d s=0 \\
& \Im\left(\underline{\boldsymbol{u}}^{\star}\right)=\int_{\mathcal{V}}\left(-\boldsymbol{\sigma}: \boldsymbol{\nabla} \underline{\boldsymbol{u}}^{\star}+\underline{\boldsymbol{f}} \cdot \underline{\boldsymbol{u}}^{\star}\right) d \mathcal{V}+\int_{\partial \mathcal{V}} \underline{\boldsymbol{t}} \cdot \underline{\boldsymbol{u}}^{\star} d \mathcal{S}=0
\end{aligned}
$$

Moreover, usual weak form of the classical diffusion equation is recalled:

$$
\Im\left(c^{\star}\right)=\int_{V}\left(\dot{c} c^{\star}-\underline{\boldsymbol{J}} \cdot \boldsymbol{\nabla} c^{\star}\right) d v+\int_{\partial V} j c^{\star} d s=0
$$


where $\phi^{\star}, c^{\star}$ and $\underline{\boldsymbol{u}}^{\star}$ respectively are an arbitrary field of virtual order parameter, concentration and displacement.

A standard Newton-Raphson's method is used to solve the system of non-linear equations in an iterative manner, where three residual vectors for the degrees of freedom $\left(\phi, c, u_{i}\right)$ and generalized stiffness matrix are calculated (Ammar et al., 2009a), following the usual rules in computational mechanics (Besson et al., 2001). An implicit Newton algorithm is used for the resolution method for global integration, based on the computation of the local consistent tangent matrix (Simo et al., 1998).

\subsection{Cahn-Larché coherent phase diagram}

This section aims at demonstrating the consequences of the choice of a specific interface behaviour on the predicted phase diagram when internal stresses are generated by transformation eigenstrains independent of concentration, typically due to lattice mismatches. Indeed, phase field models must at least be able to predict the right equilibrium conditions. This is usually not a big issue, and so it is rarely discussed in the litterature, even if subtle features can come out when mechanics is involved. For that purpose, we have chosen to test the different homogenization schemes proposed above against the well-known analytical model of (Cahn et al., 1984). For the sake of completeness, we will first recall the main features of the Cahn-Larché model; then we will compare the phase field predictions with the analytical solutions.

\subsubsection{Cahn-Larché analytical solutions}

Cahn and Larché have proposed a very simple model to exhibit some striking features of phase diagrams when elasticity is accounted for, discovered by (Williams, 1984). The simplifications allowing an explicit formulation of the equilibrium conditions are as follows:

1) The chemical free energies $f_{\alpha}$ and $f_{\beta}$ of the two coexisting phases are quadratic functions of the concentration, such as Eq. [28]. Equal curvatures $k_{\alpha}=k_{\beta}=k$ ensure that the free energies intersect only once along the concentration axis. The minima of the chemical free energies are located at $a_{\alpha}$ and $a_{\beta}\left(a_{\alpha}>a_{\beta}\right.$ by convention), which define the equilibrium concentrations in the absence of stress. Under stress, the corresponding equilibrium concentrations are different, and noted $c_{\alpha}$ and $c_{\beta}$.

2) The interfaces between $\alpha$ and $\beta$ are coherent.

3) Homogeneous isotropic linear elasticity is considered.

4) The eigenstrains are spherical tensors independent of concentration. Choosing $\beta$ as the stress free reference state, $\underset{\sim}{\varepsilon_{\beta}^{\star}}=0$ and $\underset{\sim \alpha}{\varepsilon_{\alpha}^{\star}}=\varepsilon^{\star} \underset{\sim}{\mathbf{1}}$, where $\underset{\sim}{\mathbf{1}}$ is the unit second order tensor.

5) There is no average stress.

With the four last assumptions, the elastic energy $f_{e}$ due to coherency strain is independent of the sizes, morphologies and distributions of both phases, as stated 
by the so-called Bitter-Crum theorem, see e.g. (Eshelby, 1957; Eshelby, 1959) or (Khachaturyan, 1983). $f_{e}$ depends only on the volume fraction $z$ in the following way:

$$
f_{e}=z(1-z) \mathcal{B}
$$

where $\mathcal{B}=E\left(\varepsilon^{\star}\right)^{2} /(1-\nu)$ with Young's modulus $E$ and Poisson's ratio $\nu$.

The total free energy is obtained by adding this elastic energy $f_{e}$ to the free energy of the unstressed state:

$$
f=z f_{\alpha}+(1-z) f_{\beta}+f_{e}
$$

where $z$ is the volume fraction of $\alpha$. This total free energy is supplied by the mass conservation:

$$
c_{0}=z c_{\alpha}+(1-z) c_{\beta}
$$

where $c_{0}$ is the nominal composition of the alloy. The equilibrium conditions are then obtained from the minimization of the lagrangian:

$$
\mathcal{L}=z f_{\alpha}+(1-z) f_{\beta}+f_{e}-\lambda\left[z c_{\alpha}+(1-z) c_{\beta}-c_{0}\right]
$$

where $\lambda$ is the Lagrange multiplier associated with constraint Eq. [74].

Differentiating this lagrangian with respect to $c_{\alpha}, c_{\beta}, z$ and $\lambda$ gives:

$$
\begin{aligned}
\frac{\partial \mathcal{L}}{\partial c_{\alpha}} & =z \frac{\partial f_{\alpha}}{\partial c_{\alpha}}-\lambda z=0 \\
\frac{\partial \mathcal{L}}{\partial c_{\beta}} & =(1-z) \frac{\partial f_{\beta}}{\partial c_{\beta}}-\lambda(1-z)=0 \\
\frac{\partial \mathcal{L}}{\partial z} & =f_{\alpha}-f_{\beta}+\frac{\partial f_{e}}{\partial z}-\lambda\left(c_{\alpha}-c_{\beta}\right)=0 \\
\frac{\partial \mathcal{L}}{\partial \lambda} & =z c_{\alpha}+(1-z) c_{\beta}-c_{0}=0
\end{aligned}
$$

After eliminating the Lagrange multiplier from the above equations, the conditions of coherent equilibrium can be expressed as:

$$
\begin{aligned}
\frac{\partial f_{\alpha}}{\partial c_{\alpha}}=\frac{\partial f_{\beta}}{\partial c_{\beta}} & =\mu_{\mathrm{eq}} \\
\omega_{\beta}-\omega_{\alpha} & =\frac{\partial f_{e}}{\partial z}
\end{aligned}
$$

where $\mu_{\mathrm{eq}}$ is the chemical potential at equilibrium and $\omega_{k}$ the grand potential of phase $k$ defined by $\omega_{k}=f_{k}-\mu_{\text {eq }} c_{k}$. As shown in Figure 2, Eq. [80] can be interpreted 


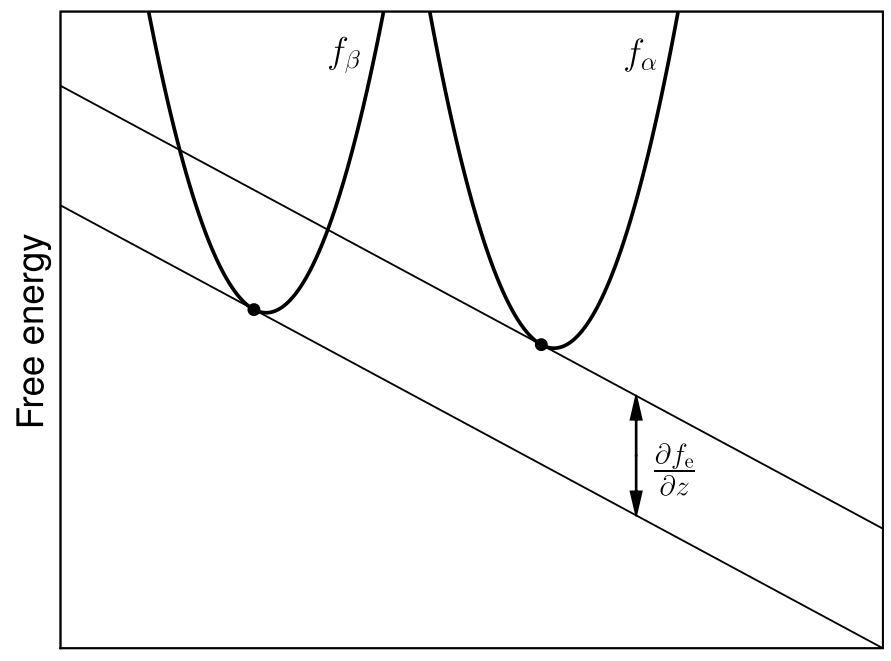

Composition $c$

Figure 2. The parallel tangent construction for determining the coexisting phase compositions for a particular value of $z$, depending on the nominal concentration $c_{0}$. The vertical gap between these parallel tangents is equal to $\partial f_{e} / \partial z$

graphically as parallel tangents to the curves $f_{\phi}$ vs $c$, when Eq. 181 quantifies the vertical gap between those tangents. When there is no elastic energy, the gap vanishes and the well-known common tangent rule is recovered.

Substituting Eqs. [80 81] into the mass conservation leads to the following expressions for $c_{\alpha}, c_{\beta}$ and $z$ :

$$
\begin{aligned}
c_{\alpha} & =a_{\alpha}+K A \\
c_{\beta} & =a_{\beta}+K A \\
z & =1 / 2-K / \Delta a
\end{aligned}
$$

where $\Delta a=a_{\alpha}-a_{\beta}, K=\left(a_{\alpha}+a_{\beta}-2 c_{0}\right) /[2(1-A)]$ and $A=2 \mathcal{B} /\left(k \Delta a^{2}\right)$.

It must be stressed that the equilibrium concentrations and the molar fraction in the phase diagram depend on the elastic energy through $A$ and on the average concentration $c_{0}$, as explicitly shown by inserting Eq. [84] in the inequality $0 \leq z \leq 1$ :

$$
a_{\beta}+\mathcal{B} /(k \Delta a) \leq c_{0} \leq a_{\alpha}-\mathcal{B} /(k \Delta a)
$$


In the particular case where $\varepsilon^{\star}$ does not follow Vegard's law, the two-phase field can shrink to a single concentration for elastic energies above a threshold called Williams' point (Williams, 1984).

All these features can be summarized on the diagram $A$ versus $c_{0}$ as shown in Figure 3 . In the absence of any elastic effects, the incoherent two-phase equilibrium is obtained and confined to a horizontal segment corresponding to $A=0$, and bounded by the equilibrium concentrations $a_{\beta}=0.3$ and $a_{\alpha}=0.7$ which are independent of the overall alloy composition $c_{0}$.

According to inequality [85], three regions can be plotted in Figure 3 Within the triangle, $\alpha$ and $\beta$ coexist coherently. The coherent two-phase region spans the ranges $0<A<1, a_{\beta}<c_{0}<a_{\alpha}$ and terminates at Williams' point $\left(c_{0}=0.5, A=1\right)$. Outside the triangle, the coherent equilibrium between $\alpha$ and $\beta$ does not occur. Then, beyond Williams' point, i.e. when $A \geq 1$, only single phase regions is obtained.

\subsubsection{Phase field calculations}

Phase field calculations using the finite element software ZeBuLon in the generalized plane strain conditions have been performed, with conditions similar to those in Cahn-Larché model: both phases are purely elastic and are attributed the same isotropic elastic moduli.

A rectangular region $(0 \leq x \leq L, 0 \leq y \leq H)$ has been meshed with linear elements, and the following conditions applied at the boundaries: vanishing order parameter flux, no mass is exchanged with surroundings, and a uniform displacement boundary condition was applied to ensure zero average stress

$$
\begin{array}{lll}
\underline{\boldsymbol{\xi}} \cdot \underline{\boldsymbol{n}}=0, \quad \underline{\boldsymbol{J}} \cdot \underline{\boldsymbol{n}}=0 & \\
u_{x}(x=0, y)=0 \quad \text { and } \quad u_{x}(x=L, y)=u_{x}(L, 0) & 0 \leq y \leq H \\
u_{y}(x, y=0)=0 & \text { and } \quad u_{y}(x, y=H)=u_{y}(0, H) & 0 \leq x \leq L
\end{array}
$$

The two boundaries (at $x=L$ and $y=H$ ) remain straight and a zero resulting force is enforced on the surface. Profiles of $\phi$ and $c$ as tanh functions along one direction have been set initially, which correspond to coexisting $\alpha$ and $\beta$ phases separated by a plane diffuse interface with a thickness roughly equal to $\delta$. This thickness has been chosen to be about one percent of the total size of the system in most of the calculations, except when specified. The evolution equations are integrated as long as the microstructure evolves with an adaptive time step.

The necessary material data used in the calculations are summarized in Table 1 All values are dimensionless and scaled with the chemical free energies curvature $k$, a mesoscopic length $L$ (typically the system size), and the characteristic time $\tau=\beta / k$ related to the interface motion. 


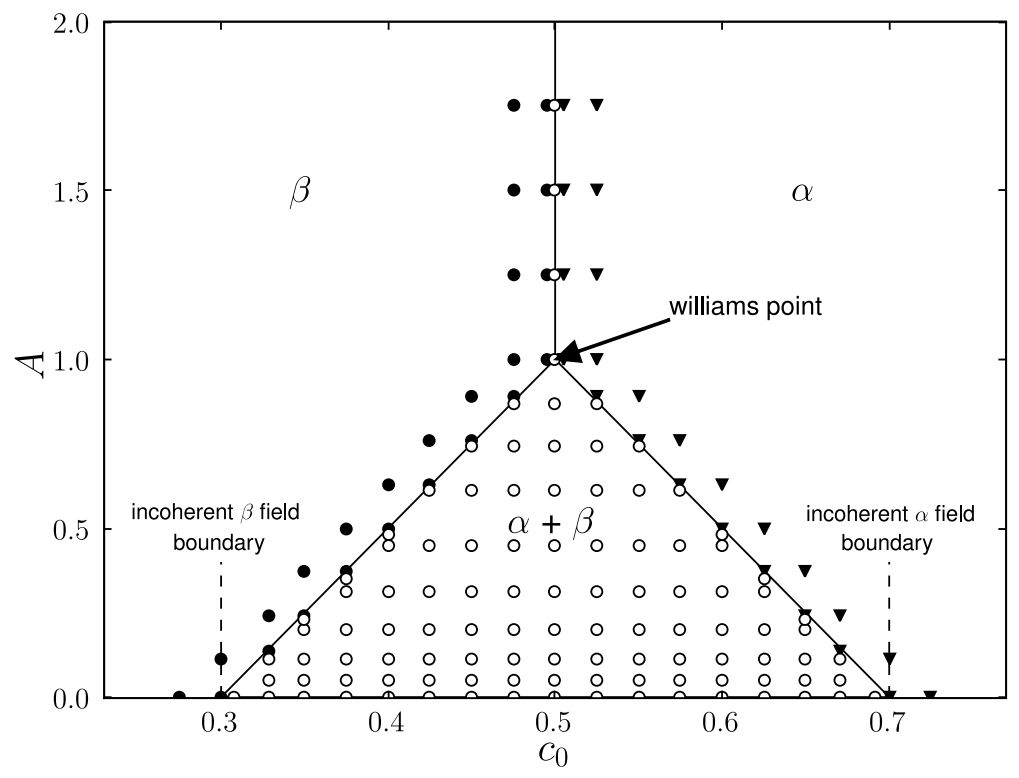

Figure 3. Coherent field diagram showing the two-phase coherent coexistence region in terms of the overall alloy concentration $c_{0}$ and the non-dimensional elastic energy A. The analytical solution of (Cahn et al., 1984) is plotted in continuous lines. The numerical results, using Voigt and Khachaturyan schemes, are plotted with $\mathbf{\nabla}, \bullet$ and $\circ$, to locate $\alpha, \beta$ and $\alpha+\beta$ phase fields respectively

Table 1. Data used in the calculations

\begin{tabular}{lcc}
\hline$E / k$ & \multicolumn{3}{c}{$7 \cdot 10^{10}$} \\
$\nu$ & \multicolumn{2}{c}{0.3} \\
$\sigma /(k L)$ & \multicolumn{2}{c}{$5 \cdot 10^{-3}$} \\
$\delta / L$ & $10^{-2}$ \\
\hline phase & $\alpha(\phi=1)$ & $\beta(\phi=0)$ \\
$a$ & 0.3 & 0.7 \\
$D \tau / L^{2}$ & 0.1 & 0.1 \\
$\sigma^{y} / k$ & - & $6 \cdot 10^{7}$ \\
$Q / k$ & - & $5 \cdot 10^{7}$ \\
$b$ & - & 10. \\
$C / k$ & - & $10^{9}$ \\
$\Gamma$ & - & 100. \\
\hline
\end{tabular}






Figure 4. Scaled elastic energy $f_{e} / k$ versus volume fraction $z$ for different diffuse interface scaled thicknesses $\delta=10^{-3}, 10^{-2}, 5 \cdot 10^{-2}$ and 0.1 . Equivalent numerical results were obtained using the various homogenization schemes

For each interface behaviour, i.e. Voigt, Reuss and Khachaturyan mixture rules, large series of calculations have been undertaken which span the two-phase field triangle and its boundaries in the diagram $A$ versus $c_{0}$, by varying the eigenstrain $\varepsilon^{\star}$ and the average concentration $c_{0}$. Each run is represented by one symbol in Figure 3 . $\mathbf{\nabla}$, - and $\circ$ when phases observed at equilibrium are $\alpha, \beta$ and $\alpha+\beta$ respectively.

As shown in Figure 3 , there is a good agreement bewteen the numerical results using Voigt and Khachaturyan schemes and the theoretical field diagram (Reuss scheme will be discussed below). However, it must be noticed that a slight discrepancy between the numerical and theoretical results appears around Williams point. This can be attributed to the finite thickness of the interface region. Indeed, increasing this thickness changes the scaling factor between $f_{e}$ and its quadratic dependence $z(1-z)$, as shown in Figure 4

The variation of the equilibrium concentrations $c_{\alpha}$ and $c_{\beta}$ with the average composition $c_{0}$ is shown in Figure 5 for different values of $A$. The numerical results obtained with Voigt/Taylor and Khachaturyan schemes are in very good agreement with the analytical solution obtained with Eq. [83]. On the contrary, Reuss scheme predicts that the compositions of the two coexisting phases are always equal to their values at incoherent equilibrium ( $c_{\beta}=0.3$ and $\left.c_{\alpha}=0.7\right)$ whatever the value of $A$. Indeed, 




Figure 5. Dependency of the equilibrium phase compositions $c_{\alpha}$ and $c_{\beta}$ on the average alloy concentration $c_{0}$ for $A=0$ (unstressed case), $A=0.1125 A=0.613$ and $A=1$ (Williams point). The continuous lines depicts the analytical solution given by Eq. (83). Symbols are for Voigt and Khachaturyan schemes. Reuss scheme is identical to these schemes with $A=0$, whatever the values of $A$

using Reuss' scheme, there can be no effect of coherency stresses on the two-phase equilibria as shown in Section 4.5 . Hence, the numerical results clearly show that this scheme is unsuitable to predict realistic coherent equilibrium conditions.

Coherent equilibria present a singular behaviour in the two-phase field: equilibrium concentrations defining the tie-lines do not coincide with the boundaries of the two-phase field. To illustrate this particularity, Figure 6 depicts how the equilibrium concentrations change with the scaled elastic energy $A$ for $c_{0}=0.55$. The numerical results obtained with Voigt and Khachaturyan schemes agree well with the analytical solution. When $\varepsilon^{\star}>0.249, \alpha$ and $\beta$ no longer coexist in coherent equilibrium and $\alpha$ is only observed. Moreover, the inability of Reuss scheme to give a consistent description of interface appears clearly in Figure 6. With this scheme, there is indeed no coupling between elastic stress and chemical equilibrium. Whatever the elastic energy, the two-phase field throughout the two-phase region, the two phases retain constant composition; $c_{\beta}=0.3$ and $c_{\alpha}=0.7$. 


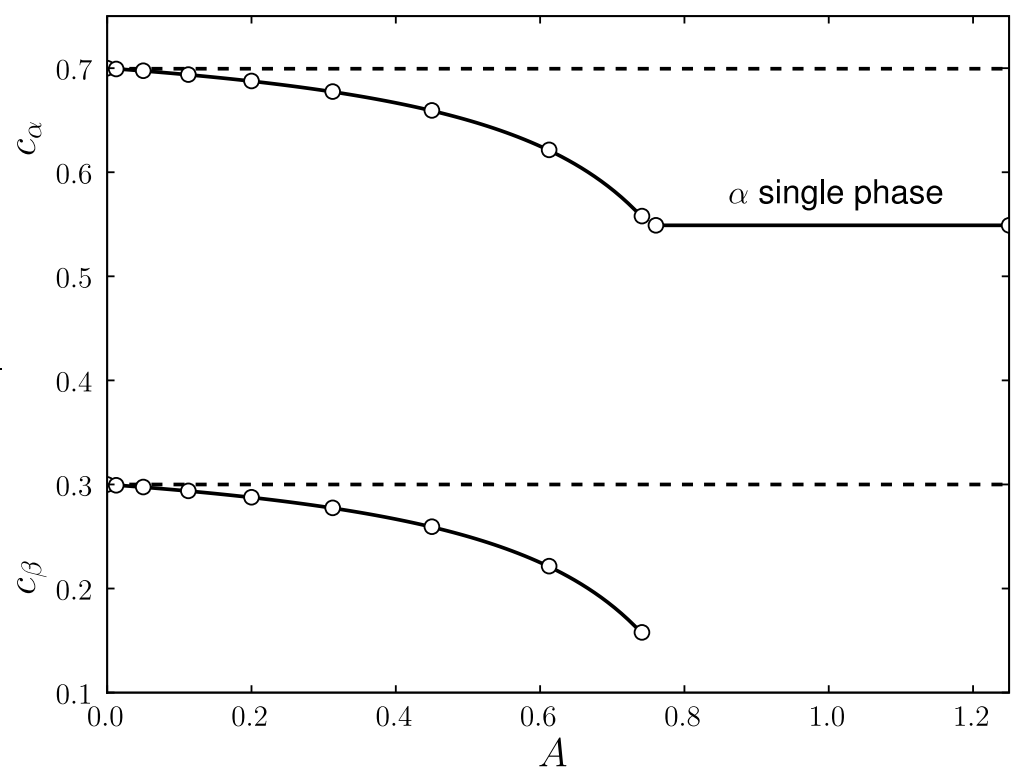

Figure 6. Equilibrium concentrations $c_{\alpha}$ and $c_{\beta}$ versus the scaled elastic energy A for $c_{0}=0.55$. The analytical solution 83 is plotted with continuous lines. Numerical results using Voigt/Taylor and Khachaturyan are with white dots. Reuss/Sachs scheme is plotted with dashed lines

\subsection{Two-phase elastoplastic alloy with hardening}

Now, we consider that the system consists of a two-phase elastoplastic binary alloy with one non-linear isotropic hardening and one non-linear kinematic hardening in each phase. The specific free energy taken as the state potential of the material is chosen as a function of all state variables. Assuming again that there is no coupling between elasticity and hardening, the free energy is split into three terms, corresponding to the elastic energy, the kinematic hardening part and the isotropic part. To satisfy the condition of thermodynamic stability, it is sufficient to choose a positive definite quadratic function in the components of elastic strain tensor and all internal state variables as follows:

$$
f_{k}=\frac{1}{2}\left(\varepsilon_{k}-\varepsilon_{k}^{\star}\right): \underset{\sim}{\boldsymbol{C}}:\left({\underset{\sim}{\varepsilon} k}_{k}-\varepsilon_{\sim}^{\star}\right)+\frac{1}{3} c_{k} \boldsymbol{\alpha}_{k}:{\underset{\sim}{\boldsymbol{\alpha}}}_{k}+\frac{1}{2} b_{k} Q_{k} r_{k}^{2}
$$

$b_{k}, Q_{k}$ and $c_{k}$ are the material parameters for isotropic and kinematic hardening states and $k=\{\alpha, \beta\}$ corresponding to the two phases (Lemaitre et al., 1994). 
A classical treatment of the Clausius-Duhem inequality provides the state laws Eqs. [44 46], defining the Cauchy stress tensor $\boldsymbol{\sigma}$ and the hardening variables $\underset{\sim}{\boldsymbol{X}}$ and $R$ in each phase:

$$
\boldsymbol{\sigma}_{k}=\underset{\approx}{\boldsymbol{C}_{k}}:\left(\varepsilon_{\sim}-\boldsymbol{\varepsilon}_{k}^{\star}-\boldsymbol{\varepsilon}_{k}^{p}\right) \quad, \quad R_{k}=b_{k} Q_{k} r_{k} \quad \text { and } \quad \underset{\sim}{\boldsymbol{X}}=\frac{2}{3} C_{k} \underset{\sim}{\boldsymbol{\alpha}_{k}}
$$

Furthermore, the mechanical dissipation is assumed to be due to three mechanisms: the inelastic strain, the kinematic hardening and the isotropic hardening. Thus, the dissipation potential can be split into a plastic contribution, which is called the yield function, a nonlinear kinematic hardening term and a nonlinear isotropic hardening term and can be expressed as a convex scalar valued function as follows (Lemaitre et al., 1994):

$$
\Omega_{u k}\left(\sigma_{k}, \underset{\sim}{\boldsymbol{X}}, R_{k}\right)=g_{k}\left(\underset{\sim}{\boldsymbol{\sigma}_{k}}, \underset{\sim}{\boldsymbol{X}}, R_{k}\right)+\frac{3 \Gamma_{k}}{4 C_{k}} \underset{\sim}{\boldsymbol{X}}: \underset{\sim}{\boldsymbol{X}} k+\frac{R_{k}^{2}}{2 Q_{k}}
$$

Assuming that the elastoplastic phase field behaviour of each phase is treated independently, we define a yield function for each phase as:

$$
g_{k}\left(\sigma_{k}, \underset{\sim}{\boldsymbol{X}}, R_{k}\right)=\sigma_{k}^{\mathrm{eq}}-R_{k}-\sigma_{k}^{y}
$$

where

$$
\sigma_{\mathrm{eq}}=\sqrt{\frac{3}{2}\left(\boldsymbol{s}_{k}-\underset{\sim}{\boldsymbol{X}_{k}}\right):\left(\boldsymbol{s}_{k}-\underset{\sim}{\boldsymbol{X}}\right)} \quad \text { where } \quad \boldsymbol{s}_{k}=\boldsymbol{\sim}_{k}-\frac{1}{3} \operatorname{Tr} \boldsymbol{\sigma}_{k} \underset{\sim}{\mathbf{1}}
$$

with $\sigma_{k}^{y}$ is the initial yield stress, $\sigma_{k}^{\text {eq }}$ is the von Mises equivalent stress and ${\underset{\sim}{k}}_{k}$ is the deviatoric stress tensor.

According to the normality rule for generalized standard materials Eqs. 4849 . [50], the evolution laws of the internal variables are derived from the dissipation potential as:

$$
\begin{aligned}
& \dot{\sim}_{k}^{p}=\frac{\partial \Omega_{u k}}{\partial g_{k}} \frac{\partial g_{k}}{\partial \boldsymbol{\sigma}_{k}}=\dot{\lambda}_{k}{\underset{\sim}{\boldsymbol{n}_{k}}} \\
& \dot{r}_{k}=\frac{\partial \Omega_{u k}}{\partial g_{k}} \frac{\partial g_{k}}{\partial R_{k}}=\dot{\lambda}_{k}\left(1-\frac{R_{k}}{Q_{k}}\right) \\
& \dot{\sim}_{k}=\frac{\partial \Omega_{u k}}{\partial g_{k}} \frac{\partial g_{k}}{\partial X_{k}}=\dot{\lambda}_{k}\left(\underset{\sim}{\boldsymbol{n}_{k}}-\frac{3 \Gamma_{k}}{2 C_{k}} \underset{\sim}{\boldsymbol{X}}\right)
\end{aligned}
$$

where ${\underset{\sim}{\boldsymbol{n}}}_{k}=\partial g_{k} / \partial \boldsymbol{\sigma}_{k}$ is the normal to the yield surface and defines the flow direction.

For phenomena which do not depend explicitly on time, such as rate independent plasticity, the potential is not differentiable. Then, the partial derivative of $\Omega_{k}$ with 
respect to $g$ is simply replaced by a plastic multiplier to write a rate independent plastic model. The plastic multiplier $\dot{\lambda}$ is determined from the consistency condition $d g_{k} / d t=0$ and $g_{k}=01$ Then, we obtain the following expression of the plastic multiplier (Benallal et al., 1988):

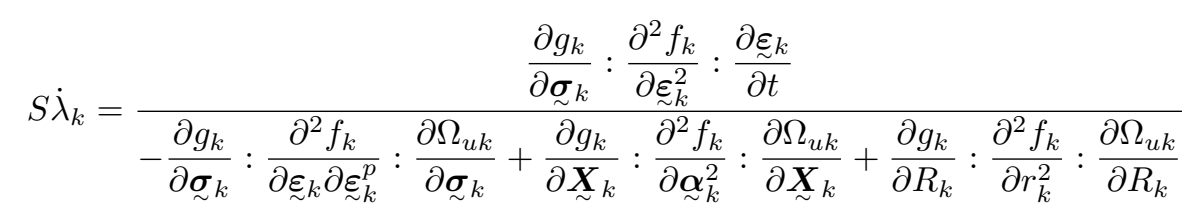

A generalized expression would be necessary in the case of a possible dependence of elastoplastic properties on concentration.

Taking the time rate of the isotropic hardening variable Eq. $\left[88 \rrbracket_{2}\right.$ and the kinematic hardening variable Eq. [88] 3 and substituting respectively into Eqs. [93] and [94], we obtain the following evolution equations:

$$
\begin{aligned}
& \dot{R}_{k}=b\left(Q_{k}-R_{k}\right) \dot{\lambda}_{k} \quad \text { equivalent to } R_{k}=Q_{k}\left(1-\exp \left(-b_{k} \lambda_{k}\right)\right) \\
& \dot{\sim}_{k}=\frac{2}{3} C_{k} \dot{\sim}_{k}^{p}-\Gamma_{k}{\underset{\sim}{\boldsymbol{X}_{k}} \dot{\lambda}_{k}}^{\dot{x}}
\end{aligned}
$$

The stress increment $\dot{\sim}_{k}$ is related to the strain increment ${\underset{\sim}{\dot{\varepsilon}}}_{k}$ by the linear elasticity law, which is classical in nonlinear mechanics (Lemaitre et al., 1994):

$$
\begin{aligned}
& \dot{\sim}_{k}=\underset{\sim}{\boldsymbol{C}} k: \stackrel{\dot{\sim}}{e}_{k}^{e} \\
& =\underset{\sim}{\boldsymbol{C}_{k}}:\left(\dot{\tilde{\varepsilon}}_{k}-\dot{\sim}_{k}^{\star}-\dot{\sim}_{k}^{p}\right)
\end{aligned}
$$

For simplicity, $\underset{\sim}{\boldsymbol{C}_{k}}$ and $\varepsilon_{k}^{\star}$ are taken here independent of concentration. After some treatment of the plãstic multiplier expression by introducing the free energy density Eq. [87] and the dissipation potential Eq. [89] and using the strain partition, in terms of rates, ${\underset{\dot{\varepsilon}}{k}}_{k}=\dot{\varepsilon}_{k}^{e}+\dot{\tilde{\varepsilon}}_{k}^{p}$, the plastic multiplier in each phase can then be written as follows:

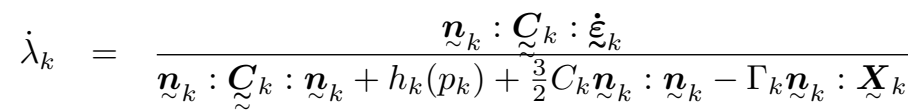

$$
\begin{aligned}
& =L_{k} \underset{\sim}{\boldsymbol{n}_{k}}:{\underset{\sim}{\boldsymbol{C}}}_{k}: \dot{\boldsymbol{\varepsilon}}_{k}
\end{aligned}
$$

1. The first condition, $g_{k}=0$, means that the state of stress is on the actual yield condition, the second $\dot{g}_{k}=0$, means that an increase of the state of stress induces an increase of the yield stress. Elastic unloading occurs when $g_{k}<0$ or $\dot{g}_{k}<0$, the internal variables then keeping a constant value. 
where

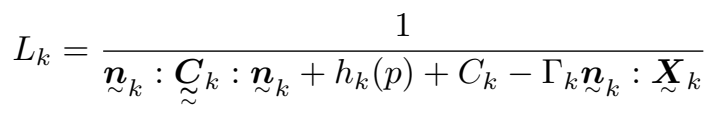

and

$$
h_{k}\left(p_{k}\right)=\frac{d R_{k}}{d r_{k}}=Q_{k} b_{k} \exp \left(-b_{k} r_{k}\right)
$$

Using Voigt's model, the total strain rate is assumed to be the same for both phases, according to Eq. $[51]_{2}$ :

$$
\dot{\sim}_{\alpha}=\dot{\sim}_{\beta}=\underset{\sim}{\dot{\varepsilon}}
$$

According to Reuss model, the total strain rate in each phase is no longer equal to the local total strain rate. After some manipulations, the total strain rate for the $\alpha$ and $\beta$ phases can be formulated, on the basis of the properties and variables related to the different phases, as (details are provided in Appendix A2)

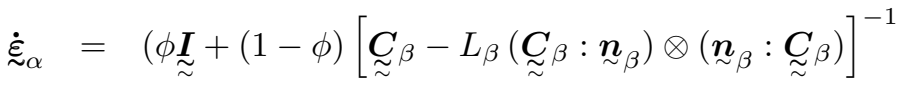

$$
\begin{aligned}
& \left.\cdot\left(\underset{\sim}{\boldsymbol{C}} \alpha-L_{\alpha}(\underset{\sim}{\boldsymbol{C}} \alpha: \underset{\sim \alpha}{\boldsymbol{n}}) \otimes(\underset{\sim}{\boldsymbol{n}}: \underset{\sim}{\boldsymbol{C}} \alpha)\right)\right)^{-1}:\left(\underset{\sim}{\dot{\varepsilon}}-\dot{\phi}\left(\underset{\sim}{\boldsymbol{\varepsilon}} \alpha-{\underset{\sim}{\boldsymbol{\varepsilon}} \beta}_{\beta}\right)\right) \\
& \dot{\sim}_{\beta}=\left((1-\phi) \underset{\approx}{\boldsymbol{I}}+\phi\left[\underset{\approx}{\boldsymbol{C}} \alpha-L_{\alpha}\left(\underset{\approx}{\boldsymbol{C}}:_{\sim \alpha}\right) \otimes(\underset{\sim \alpha}{\boldsymbol{n}}: \underset{\approx}{\boldsymbol{\boldsymbol { n }}} \alpha)\right]^{-1}\right. \\
& \left.\cdot\left(\underset{\sim}{\boldsymbol{C}} \beta-L_{\beta}(\underset{\sim}{\boldsymbol{C}} \beta: \underset{\sim}{\boldsymbol{n}}) \otimes(\underset{\sim}{\boldsymbol{n}}: \underset{\sim}{\boldsymbol{\sim}} \beta)\right)\right)^{-1}:\left(\underset{\sim}{\dot{\varepsilon}}-\dot{\phi}\left({\underset{\sim}{\varepsilon} \alpha}_{\alpha}-{\underset{\sim}{\boldsymbol{\varepsilon}} \beta}_{\beta}\right)\right)
\end{aligned}
$$

These formula are needed for the implementation of the Reuss/Sachs procedure.

\subsection{Growth of an elastoplastic particle in an elastic matrix}

In order to investigate the effect of plastic accommodation on the transformation kinetics and plastic strain distribution, 2D finite element calculations have been performed considering the growth of an elastoplastic precipitate growing in an elastic matrix. The $\beta$ matrix is considered to behave in a purely elastic way. Hence plastic deformation can occur only in the $\alpha$ precipitate adjacent to the matrix. One considers one non-linear isotropic hardening and one non-linear kinematic hardening in the elastoplatic phase. The growth of the cylindrical $\alpha$ precipitate in a cylindrical matrix $\beta$ is simulated under generalized plane strain conditions. The calculation has been performed using the Voigt/Taylor model, since Reuss model has been shown to be inappropriate. 
The finite element mesh is composed of quadratic 8-nodes quadrangular elements, as shown in Figure 7. The following boundary conditions to the system have been applied:

$$
\begin{array}{lll}
\underline{\boldsymbol{\xi}} \cdot \underline{\boldsymbol{n}}=0, & \underline{\boldsymbol{J}} \cdot \underline{\boldsymbol{n}} & \text { on all boundaries } \\
\underset{\boldsymbol{\sigma}}{(r}=R, \theta)=0 & 0 \leq \theta \leq \theta_{0} & : \text { free surface condition } \\
u_{\theta}(r, \theta=0)=0 & 0 \leq r \leq R & \\
u_{\theta}\left(r, \theta=\theta_{0}\right)=0 & 0 \leq r \leq R & : \text { symmetric boundary condition }
\end{array}
$$

The lower and upper boundaries at $\theta=0$ and $\theta=\theta_{0}$ remain straight. Radial profiles as tanh funtion has been imposed initially for $\phi$ and $c$, corresponding to the presence of a cylindrical $\alpha$ precipitate in the $\beta$ shell matrix with an initial radius $x_{0} / R=0.5$, where $R$ is the total radius of the cylindrical system. This initial condition ensures that the precipitate will grow, at least in a pure elastic case, according to Cahn-Larché coherent diagram. Finally, we have kept the chemical and elastic parameters reported in Table 1

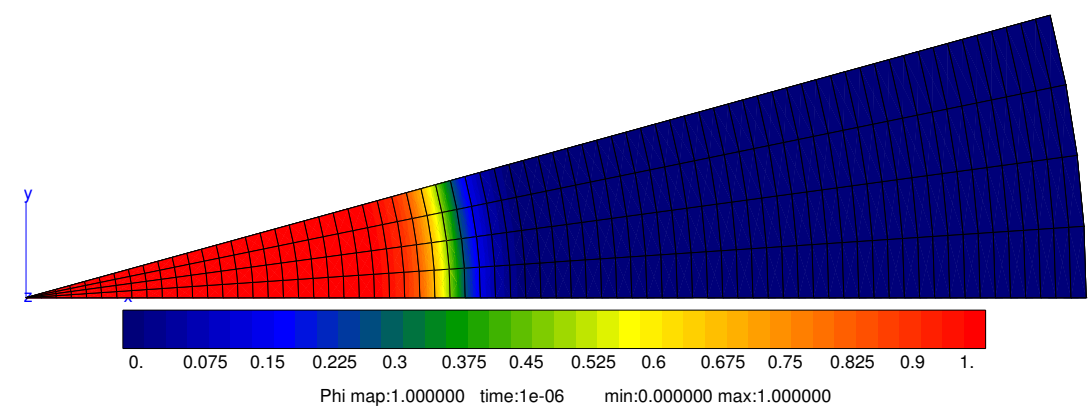

Figure 7. Initial phase field following a variation in tanh along the cylinder radius in a $2 D$ finite element mesh

As shown in Figure 8 with the vertical dotted lines corresponding to the level set $\phi=0.5$, the interface moves forwards to higher values of $x$ : the precipitate grows at the expense of the $\beta$ matrix. The growth is mainly driven by the diffusion of the alloying species from the matrix to the interface as shown in Figure 8 . As the transformation proceeds, the supersaturation in alloying species of the $\beta$ matrix decreases to zero when the equilibrium concentration $c_{\beta}$ is reached. As shown in Figure $8 \mathrm{~b}$ for $\sigma_{\theta \theta}$ component, stresses develop inside the matrix. Interacting with the free boundary, the equivalent stress decreases within the precipitate, but increases in the interface on the side abutting on the $\beta$ matrix. At this very location, the equivalent stress progressively reaches the yield strength and plasticity starts in the interface, after about 50 time steps. The normalized equivalent effective plastic strain $E_{\mathrm{eq}}^{p} / E_{\text {eqmax }}^{p}$ is shown 

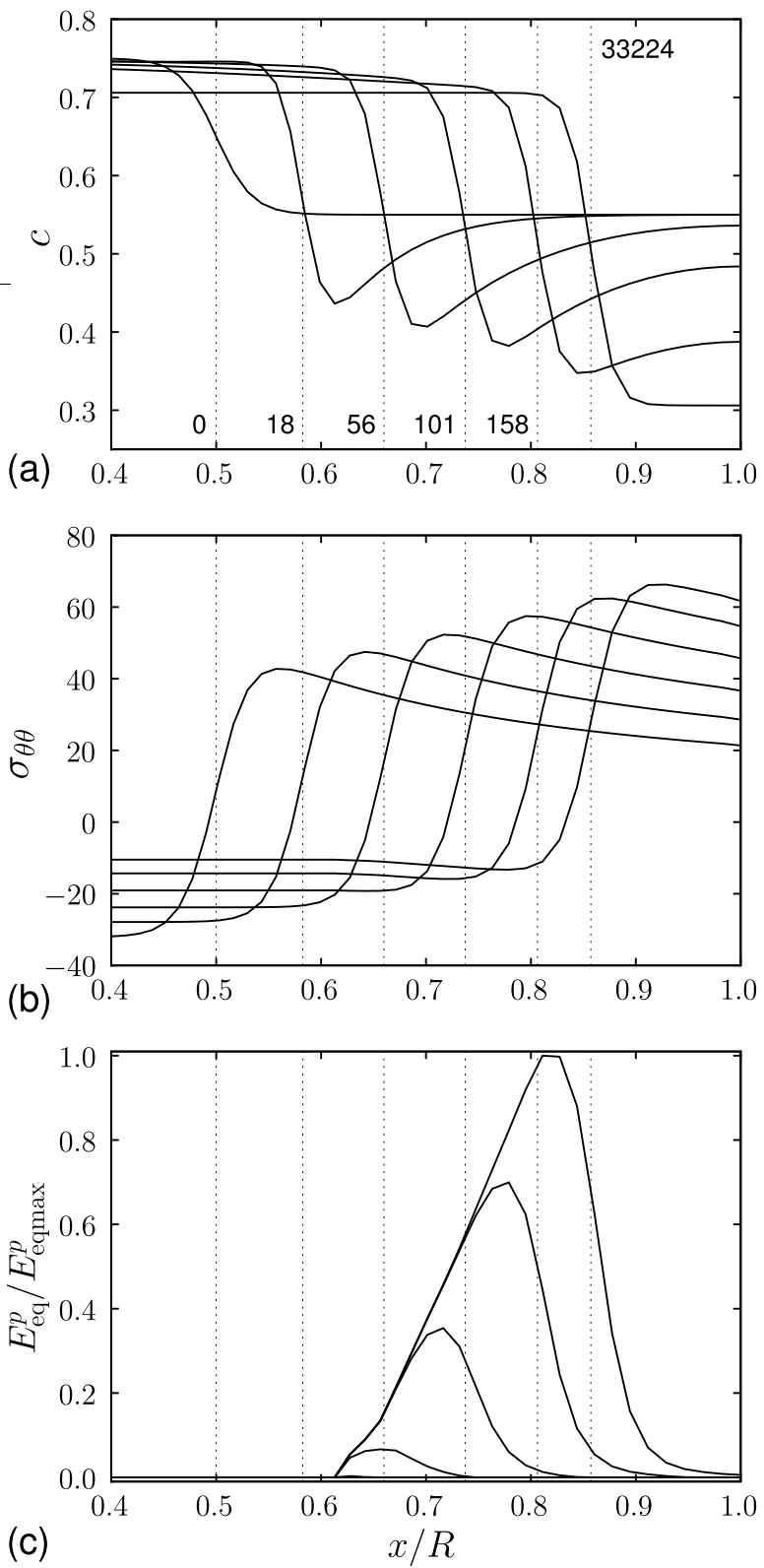

Figure 8. Time evolution of the (a) concentration field, $(b)$ stress component $\sigma_{\theta \theta}$ and (c) normalized equivalent effective plastic strain $E_{\mathrm{eq}}^{p} / E_{\mathrm{eqmax}}^{p}$, for $\varepsilon^{\star}=3 \cdot 10^{-4}$ and $E_{\text {eqmax }}^{p}=2.28 \cdot 10^{-5}$, using Taylor model. Dotted lines locate the successive positions of the interface at different the time steps indicated in (a) 
at different time steps in Figure 8p, where the equivalent effective plastic strain is defined as:

$$
E_{\mathrm{eq}}^{p}=\sqrt{\frac{2}{3} \mathcal{\sim}^{p}:{\underset{\sim}{\varepsilon^{p}}}^{p}}
$$

where $E_{\text {eqmax }}^{p}$ is the maximal equivalent plastic strain when the interface is at $x / R=$ 0.86 , end of our computation.

At the end of the transformation, a peak of equivalent plastic strain is observed due to its progressive build-up at the moving interface. The presence of this peak is a particular feature of the process studied, where the mechanical interaction with the external boundary is significant.

\section{Conclusions}

A general constitutive framework has been proposed to incorporate, in a systematic way, linear and nonlinear mechanical behaviour laws into a phase field model. The systematic procedure can be summarized as follows:

1) Select a diffusion/phase field model with its corresponding free energy and dissipation potentials for the chemical processes. In the examples given here, we have retained a variant of the approach proposed by (Kim et al., 1998).

2) Select for each individual phase a set of mechanical constitutive equations. Following the standard generalized framework settled in (Germain et al., 1983; Lemaitre et al., 1994), the mechanical part of the free energy and dissipation potentials can be chosen. The nonlinear behaviour is accounted for by means of appropriate internal variables. In the present approach, the mechanical properties and parameters associated with each phase may depend on concentrations, exclusively, but not on the order parameter.

3) Attach to each material point stress and strain tensors associated with each considered phase, that are related to the classical stress and strain at that point by the following formula:

$$
\begin{aligned}
\underset{\sim}{\boldsymbol{\varepsilon}} & =\chi \underset{\sim}{\boldsymbol{\varepsilon}_{\alpha}}+(1-\chi) \boldsymbol{\sim}_{\beta} \\
\underset{\sim}{\boldsymbol{\sigma}} & =\chi \boldsymbol{\sim}_{\alpha}+(1-\chi) \boldsymbol{\sigma}_{\beta} \\
f_{u} & =\chi f_{\alpha u}+(1-\chi) f_{\beta u}
\end{aligned}
$$

where the indicator function $\chi$ is a function of order parameter. In the examples provided in this work, we take:

$$
\chi=\phi
$$

4) Select a homogenisation scheme in order to determine the previous variables $\varepsilon_{\alpha, \beta}, \sigma_{\alpha, \beta}$ for given stress and strain values $\underset{\sim}{\boldsymbol{\sigma}} \boldsymbol{\sigma}$ at each material point. This scheme also implies specific definitions of the effective stiffess, eigenstrain and plastic deformation at each material point, which are especially important in the diffuse interface 
zone. According to the explored Voigt model, the effective stiffness is the arithmetic mean of the stiffness tensors of the individual phases. The effective eigenstrains, including plastic strain, are not given by such simple means. The correct relations have been provided. According to Reuss' approach, the effective compliance and the effective eigenstrains are arithmetic means of the corresponding values of the phases.

The performed analysis has shown that the usual interpolation rules used in microelastic phase field models combine aspects of both Reuss and Voigt approaches in the sense that they take simultaneously arithmetic mean values for elastic moduli and eigenstrains. However, it turns out that using these standard interopolation rules and the full Voigt/Taylor model lead to the same results in terms of equilibrium concentrations under eigenstresses, and also for the growth of an elastic-plastic cylindrical precipitate in an elastic matrix, with the same elastic moduli. In contrast, the Reuss procedure has been shown to be inadequate since it does not allow enough influence of mechanics on diffusion.

Combining different homogenization schemes is acceptable since there is no clear physical motivation for selecting one or another in the present context as long as no specific hypothesis is made on the atomic arrangement inside the representative volume element underlying each material point, and as long as no specific discretecontinuum homogenization scheme is constructed. Other general homogenization schemes could be tested and may be computationally more efficient under circumstances, like the Hashin-Shtrikman procedure or the self-consistent method.

Applications of the proposed theory will deal with the case of equilibrium concentrations in the presence of heterogeneous elastic behaviour and then in the presence of plastic deformation, which are still poorly known fields of phase transformation.

In particular, the question of inheritance of plastic deformation during migration of phase boundaries has not been addressed in this work. It remains to be explored in order to reach realistic comparison with experimental results.

\section{References}

Ammar K., Appolaire B., Cailletaud G., Feyel F. andForest S., " Finite element formulation of a phase field model based on the concept of generalized stresses", Computational Materials Sciences, vol. 45, p. 800-805, 2009a.

Ammar K., Appolaire B., Cailletaud G., Feyel F. andForest S., " Finite element formulation of an elastoplastic phase-field model for binary alloys based on the concepts of generalized stresses and homogenization methods", Plasticity 2009, p. 3-6, 2009 b.

Ammar K., Appolaire B., Cailletaud G., Feyel F., Forest S., “Formulation éléments finis des modèles de champ de phases basée sur la théorie de l'équilibre des microforces", Plast0x 2007, EDP Sciences, p. 117-129, 2009c.

Appolaire B., Aeby-Gautier E., Da Costa Teixeira J., Dehmas M., Denis S., “ Non coherent interfaces in diffuse interface models", Philosophical Magazine, 2009. in presse. 
Appolaire B., Gautier E., “ Modelling of phase transformations in titanium alloys with a phase field model”, Lecture Notes in Computational Science \& Engineering, vol. 32, p. 196-201, 2003.

Barbe F., Quey R., Taleb L., Souza de Cursi E., “ Numerical modelling of the plasticity induced during diffusive transformation. An ensemble averaging approach for the caseof random arrays of nuclei”, European Journal of Mechanics A/Solids, vol. 27, p. 1121-1139, 2008.

Benallal A., Billardon R., Doghri I., “ An integration algorithm and the corresponding consistent tangent operator for fully coupled elastoplastic and damage equations", Comm. Appl. Num. Meth., vol. 4, p. 731-740, 1988.

Besson J., Cailletaud G., Chaboche J.-L., Forest S., Mécanique non linéaire des matériaux, Hermès Sciences, France, 2001.

Boussinot G., Finel A., Le Bouar Y., “ Phase-field modeling of bimodal microstructures in nickel-based superalloys”, Acta Materialia, vol. 57, p. 921-931, 2009.

Cahn J., Larche F., “ A simple model for coherent equilibrium”, Acta Metall., vol. 32, n ${ }^{\circ} 11$, p. 1915-1923, 1984.

Cha P.-R., Kim J., Kim W.-T., Kim S., “ Effect of transformation induced stress and plastic deformation on austenite/ferrite transition in low carbon steel", Plasticity 2009, p. 376-378, 2009.

Coleman B., Gurtin M., “ Thermodynamics with internal variables”, The Journal of Chemical Physics, vol. 47, p. 597-613, 1967.

Coleman B., Noll W., “ The thermodynamics of elastic materials with heat conduction andviscosity”, Arch. Rational Mech. and Anal., vol. 13, p. 167-178, 1963.

Eiken J., Böttger B., Steinbach I., “ Multiphase-field approach for multicomponent alloys with extrapolation scheme for numerical application", Physical Review E, vol. 73, p. 066122-1-9, 2006.

Eshelby J., " The determination of the elastic field of an ellispoidal inclusion, and related problems", Proceedings of the Royal Society of London. Series A, vol. 241, p. 376-396, 1957.

Eshelby J., “ The elastic field outside an ellipsoidal inclusion”, Proceedings of the Royal Society of London. Series A, vol. 252, p. 561-569, 1959.

Foerch R., Besson J., “Large scale object oriented finite element code design”, Comput. Methods Appl. Mech. Eng., vol. 142, p. 165-187, 1997.

Forest S., "The micromorphic approach to plasticity and diffusion”, in D. Jeulin, S. Forest (eds), Continuum Models and Discrete Systems 11, Proceedings of theinternational conference CMDS11, Les Presses de l'Ecole des Mines de Paris, Paris, France, p. 105-112, 2008.

Forest S., " The micromorphic approach for gradient elasticity, viscoplasticity and damage", ASCE Journal of Engineering Mechanics, vol. 135, p. 117-131, 2009.

Ganghoffer J., Simonsson K., Denis S., Gautier E., Sjöström S., Simon A., “ Martensitic transformation plasticity simulations by finite elements", Journal de Physique IV (France), vol. 4, p. C3-215-220, 1994.

Gaubert A., Finel A., Le Bouar Y., Boussinot G., " Viscoplastic phase field modellling of rafting in Ni base superalloys", Continuum Models and Discrete Systems CMDS11, Mines Paris Les Presses, p. 161-166, 2008.

Gaubert A., Le Bouar Y., Finel A., " Coupling phase field and visco-plasticity to study rafting in Ni-base superalloys”, Philosophical Magazine, 2009. 
Germain P., Nguyen Q.-S., Suquet P., “Continuum Thermodynamics”, J. Appl. Mech., vol. 50, p. 1010-1020, 1983.

Guo X., Shi S., Ma X., “Elastoplastic phase field model for microstructure evolution”, Applied Physics Letters, vol. 87, p. 221910-1-3, 2005.

Guo X., Shi S., Zhang Q., Ma X., “ An elastoplastic phase-field model for the evolution of hydride precipitation in zirconium, Part I: smooth specimens", Journal of Nuclear Materials, vol. 378, p. 110-119, 2008.

Gurtin M., "Generalized Ginzburg-Landau and Cahn-Hilliard equations based on a microforce balance", Physica D, vol. 92, p. 178-192, 1996.

Jeulin D., Ostoja-Starzewski M., Mechanics of Random and Multiscale Microstructures, CISM Courses and Lectures No. 430, Udine, Springer Verlag, 2001.

Khachaturyan A., Theory of Structural Transformations in Solids, John Wiley \& Sons, New York, 1983.

Kim S., Kim W., Suzuki T., “ Interfacial compositions of solid and liquid in a phase-field model with finite interface thickness for isothermal solidification in binary alloys", Physical Review E, vol. 58, n 3, p. 3316-3323, 1998.

Kim S., Kim W., Suzuki T., “Phase-field model for binary alloys”, Physical Review E, vol. 60, n 6, p. 7186-7197, 1999.

Le bouar Y., Loiseau A., Khachaturyan A., " Origin of chessboard-like structures in decomposing alloys. Theoretical model and computer simulation”, Acta Metallurgica, vol. 46, $\mathrm{n}^{\circ} 8$, p. 2777-2788, 1998.

Lemaitre J., Chaboche J.-L., Mechanics of Solid Materials, University Press, Cambridge, UK, 1994.

Nakajima K., Apel M., Steinbach I., " The role of carbon diffusion in ferrite on the kinetics of cooperative growth of pearlite: A multi-phase field study", Acta Materialia, vol. 54, p. 3665-3672, 2006.

Nemat-Nasser S., Hori M., Micromechanics: Overall Properties of heterogeneous Solids, $2^{\text {nd }}$ edition edn, Elsevier Science Publishers, 1999.

Onuki A., " Ginzburg-Landau Approach to Elastic Effects in the Phase Separation of Solids", Journal of the Physical Society of Japan, vol. 58, p. 3065-3068, 1989.

Qu J., M. C., Fundamentals of micromechanics of solids, John Wiley \& Sons Inc, Hoboken, 2006.

Rodney D., Le bouar Y., Finel A., " Phase field methods and dislocations”, Acta Materialia, vol. 51, p. 17-30, 2003.

Simo J., Hughes T., Computational inelasticity, Springer Verlag, New York, 1998.

Steinbach I., Apel M., “ Multi phase field model for solid state transformation with elastic strain”, Physica D, vol. 217, p. 153-160, 2006.

Steinbach I., Apel M., “ The influence of lattice strain on pearlite formation in Fe-C”, Acta Materialia, vol. 55, p. 4817-4822, 2007.

Suquet P., Continuum micromechanics, CISM Courses and Lectures No. 377, Udine, Springer Verlag, Berlin, 1997.

T. Uehara T., Tsujino T., Ohno N., “ Elasto-plastic simulation of stress evolution during grain growth using a phase field model”, Journal of Crystal Growth, vol. 300, p. 530-537, 2007. 
Ubachs R., Schreurs P., Geers M., " A nonlocal diffuse interface model for microstructure evolution of tin-lead solder", Journal of the Mechanics and Physics of Solids, vol. 52, p. 1763-1792, 2004.

Ubachs R., Schreurs P., Geers M., " Phase field dependent viscoplastic behaviour of solder alloys", International Journal of Solids and Structures, vol. 42, p. 2533-2558, 2005.

Wang Y., Chen L.-Q., Khachaturyan A., " Kinetics of strain-induced morphological transformation in cubic alloys with a miscibility gap", Acta Metallurgica et Materialia, vol. 41, p. 279-296, 1993.

Williams R. O., “ The Calculation of Coherent Phase Equilibria”, Calphad, vol. 8, n 1, p. 1-14, 1984.

Yamanaka A., Takaki T., Tomita Y., “ Elastoplastic phase-field simulation of self- and plastic accomodations in cubic $\rightarrow$ tetragonal martensitic transformation", Materials Science \& Engineering A, vol. 491, p. 378-384, 2008.

Zhou N., Shen C., Mills M., Wang Y., " Contributions from elastic inhomogeneity and from plasticity to $\gamma^{\prime}$ rafting in single-crystal Ni-Al”, Acta Materialia, vol. 56, p. 6156-6173, 2008.

\section{Appendix A1. Notation}

The following symbols are used in this paper:

Symbol
$a_{\alpha}, a_{\beta}$
$A_{k}$
$c, c_{\alpha}, c_{\beta}$
$c_{0}$
$c^{\star}$
$\underset{\widetilde{D}}{\boldsymbol{C}}, \boldsymbol{\sim}_{\alpha},{\underset{\approx}{\beta},}_{\approx} \boldsymbol{\sim f f f}$
$D_{\phi}$
$D_{c}$
$D_{u}$
$D_{\alpha}, D_{\beta}$
$\mathcal{D}$
$E, E_{\alpha}, E_{\beta}$
$e$
$\mathcal{E}$
$F$
$f, f_{\alpha}, f_{\beta}$
$f_{\mathrm{ch}}$
$f_{e}, f_{e \alpha}, f_{e \beta}$
$f_{p}, f_{p \alpha}, f_{p \beta}$
$f_{u}$

Meaning

Equilibrium concentrations at incoherent state Thermodynamical force associated with internal variable $V_{k}$ Coherent equilibrium concentrations

Initial concentration

Virtual concentration

Fourth-order tensor of elasticity moduli

Residual dissipation

Phase field dissipation

Chemical dissipation

Mechanical dissipation

Chemical diffusivities

Subdomain of body

Young's moduli

Internal energy density

Internal energy of the body

Total free energy

Free energy densities

Chemical free energy density

Elastic free energy densities

Plastic free energy densities

Mechanical free energy density 


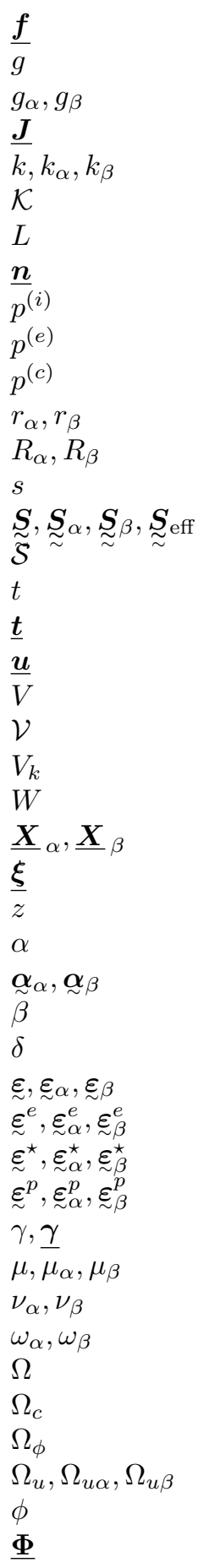

Volume force density

Double well potential

Yield function

Diffusion flux

Curvature of the free energy density

Kinetic energy

Onsager coefficient

Normal unit vector

Virtual power density of internal forces

Virtual power density of long range volume forces

Virtual power density of generalized contact forces

Scalar variables of isotropic hardening

Isotropic hardening variables

Entropy density

Fourth-order compliance tensors

Area

Time

Surface density of cohesion forces

Displacement field

Volume

Material representative volume element

Internal variable

Height of double-well barrier

Tensor variables of kinematic hardening

Vector microstress

Volume fraction

Composition gradient energy coefficient

Kinematic hardening variables

Material parameter related to the interface mobility Interfacial thickness

Total strains

Elastic strains

Eigenstrains

Plastic strains

Scalar and vector external microforces

Chemical potentials

Poisson's ratios

Grand potentials

Dissipation potential

Chemical dissipation potential

Phase field dissipation potential

Mechanical dissipation potentials

Order parameter

Entropy flux 


$\begin{array}{ll}\pi & \text { Scalar microstress } \\ \pi_{\text {dis }} & \text { Chemical force associated with the dissipative processes } \\ \underset{\sim}{\boldsymbol{\sigma}}, \boldsymbol{\sigma}_{\alpha}, \boldsymbol{\sigma}_{\beta} & \text { Cauchy stress tensor } \\ \sigma & \text { Interfacial energy } \\ \sigma_{\alpha}^{\mathrm{eq}}, \sigma_{\beta}^{\mathrm{eq}} & \text { Von Mises equivalent stress } \\ \sigma_{\alpha}^{y}, \sigma_{\beta}^{y} & \text { Initial yield stress } \\ \zeta & \text { Surface density of microtraction }\end{array}$

\section{Appendix A2. Expression of the total strain rate tensors for both phases}

According to Reuss/Sachs' scheme, we have, at each material point:

$$
\dot{\boldsymbol{\sigma}}=\dot{\sim}_{\alpha}=\dot{\boldsymbol{\sigma}}_{\beta}
$$

The linear elastic law is applied for each phase, taking the partition hypothesis of strain into account:



The substitution of the plastic multiplier [98] into [92] leads to the evolution equation of the plastic strain:

$$
\dot{\boldsymbol{\varepsilon}}_{k}^{p}=\left(L_{k} \underset{\sim}{\boldsymbol{n}_{k}}: \underset{\sim}{\boldsymbol{C}_{k}}: \dot{\boldsymbol{\varepsilon}}_{k}\right) \boldsymbol{n}_{k}
$$

where $L_{k}$ is given by [99].

After combining Eq.[111] and Eq.[112], we obtain, therefore:

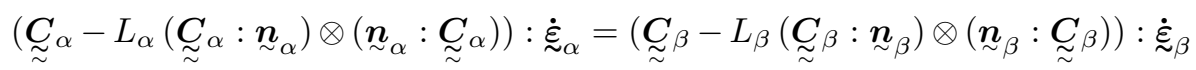

Consequently, we deduce the following relation, which relates the total strain rates of both phases $\underset{\sim_{\alpha}}{\dot{\varepsilon}_{\alpha}}$ and ${\underset{\sim}{\xi}}_{\beta}$ :

$$
\begin{aligned}
& \dot{\sim}_{\beta}=\left[\underset{\approx}{\boldsymbol{C}} \beta-L_{\beta}(\underset{\sim}{\boldsymbol{C}} \beta: \underset{\sim \beta}{\boldsymbol{n}}) \otimes(\underset{\sim}{\boldsymbol{n}}: \underset{\sim}{\boldsymbol{C}} \beta)\right]^{-1} \\
& \cdot\left(\underset{\sim}{\boldsymbol{C}} \alpha-L_{\alpha}\left(\underset{\sim}{\boldsymbol{C}}: \underset{\sim \alpha}{\boldsymbol{n}_{\alpha}}\right) \otimes(\underset{\sim \alpha}{\boldsymbol{n}}: \underset{\approx}{\boldsymbol{C}})\right): \dot{\boldsymbol{\varepsilon}}_{\alpha}
\end{aligned}
$$

Moreover, the average strain rate is then written in the following form:

$$
\begin{aligned}
& \underset{\sim}{\dot{\boldsymbol{\varepsilon}}}=\phi \underset{\sim}{\dot{\boldsymbol{\varepsilon}}_{\alpha}}+(1-\phi) \dot{\sim}_{\beta}+\dot{\phi}\left(\boldsymbol{\varepsilon}_{\alpha}-\boldsymbol{\varepsilon}_{\beta}\right)
\end{aligned}
$$

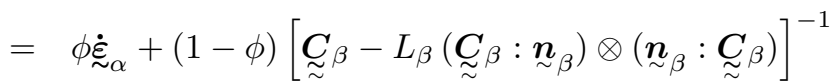

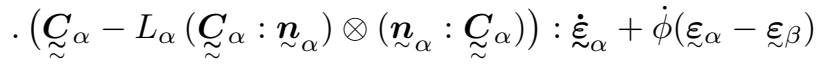


Consequently, the total strain rates in $\alpha$ and $\beta$ phases are obtained as functions of the total strain rates and the current strain states in each phase:

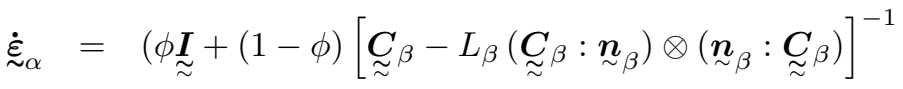

$$
\begin{aligned}
& \text {. } \left.\left(\underset{\sim}{\boldsymbol{C}} \alpha-L_{\alpha}(\underset{\sim}{\boldsymbol{C}} \alpha: \underset{\sim}{\boldsymbol{n}}) \otimes(\underset{\sim \alpha}{\boldsymbol{n}}: \underset{\approx}{\boldsymbol{C}} \alpha)\right)\right)^{-1}:\left(\underset{\sim}{\dot{\varepsilon}}-\dot{\phi}\left({\underset{\sim}{\boldsymbol{\varepsilon}} \alpha}_{\alpha}-\underset{\sim}{\boldsymbol{\varepsilon}}\right)\right) \\
& \dot{\sim}_{\beta}=\left((1-\phi) \underset{\sim}{\boldsymbol{I}}+\phi\left[\underset{\sim}{\boldsymbol{C}} \alpha-L_{\alpha}(\underset{\sim}{\boldsymbol{C}} \alpha: \underset{\sim \alpha}{\boldsymbol{n}}) \otimes(\underset{\sim \alpha}{\boldsymbol{n}}: \underset{\sim}{\boldsymbol{C}} \alpha)\right]^{-1}\right. \\
& \left..\left(\underset{\sim}{\boldsymbol{C}} \beta-L_{\beta}\left(\underset{\sim}{\boldsymbol{C}} \beta: \underset{\sim}{\boldsymbol{n}_{\beta}}\right) \otimes\left(\underset{\sim}{\boldsymbol{n}_{\beta}}: \underset{\sim}{\boldsymbol{C}_{\beta}}\right)\right)\right)^{-1}:\left(\underset{\approx}{\dot{\varepsilon}}-\dot{\phi}\left(\varepsilon_{\alpha}-\varepsilon_{\beta}\right)\right)
\end{aligned}
$$

Received: 6 May 2009 Accepted: 8 October 2009 
